\title{
Very general holographic superconductors and entanglement thermodynamics
}

\author{
Anshuman Dey, Subhash Mahapatra and Tapobrata Sarkar \\ Department of Physics, Indian Institute of Technology, \\ Kanpur 208016, India \\ E-mail: deyanshu@iitk.ac.in, subhmaha@iitk.ac.in, tapo@iitk.ac.in
}

\begin{abstract}
We construct and analyze holographic superconductors with generalized higher derivative couplings, in single R-charged black hole backgrounds in four and five dimensions. These systems, which we call very general holographic superconductors, have multiple tuning parameters and are shown to exhibit a rich phase structure. We establish the phase diagram numerically as well as by computing the free energy, and then validated the results by calculating the entanglement entropy for these systems. The entanglement entropy is shown to be a perfect indicator of the phase diagram. The differences in the nature of the entanglement entropy in R-charged backgrounds compared to the AdS-Schwarzschild cases are pointed out. We also compute the analogue of the entangling temperature for a subclass of these systems and compare the results with non-hairy backgrounds.
\end{abstract}

KEYWORDS: Holography and condensed matter physics (AdS/CMT), Gauge-gravity correspondence, AdS-CFT Correspondence

ArXiv EPRINT: 1409.5309 


\section{Contents}

1 Introduction 1

2 4-D R-charged black hole backgrounds $\quad 6$

3 5-D R-charged black hole backgrounds 13

4 HEE for very general holographic superconductors $\quad 15$

$5 \quad$ Entangling temperature of holographic superconductors 21

6 Conclusions and discussions $\quad 25$

A Details of 5-D single R-charged black hole backgrounds 26

B Necessary formulas for the VGHS in 4-D AdS-Schwarzschild backgrounds 28

\section{Introduction}

It is by now well recognized that holographic AdS/CFT duality [1] can provide valuable insights into the physics of strongly coupled systems which may not be amenable to a perturbative analysis. This duality, which relates a classical theory of AdS gravity to a conformal field theory in one lower dimension (living on the boundary of the AdS space) has in particular found important applications in the study of strongly coupled condensed matter systems. Although it is fair to say that connections to realistic condensed matter physics via the holographic correspondence has so far remained elusive, it is important to explore this line of research and further our understanding towards the ultimate goal of connecting to experimental results.

Two of the most important aspects that have received wide attention in the context of the AdS/CFT correspondence are holographic superconductors, initiated by the works of [2-4] and holographic entanglement entropy (HEE), introduced in [5]. While we expect that the former might capture important physical effects in realistic superconducting systems, the latter should be of importance in, for example, areas related to information theory. Several authors have, in the recent past, studied various aspects of holographic superconductors and in particular, HEE in that setting [6].

In a previous work [7], we had built a model of a generalized holographic superconductor, with a generalized form of higher derivative couplings, following the work of $[8,9]$. To our knowledge this is the most general phenomenological model of a holographic superconductor constructed till date, and shows rich phase structure compared to other models considered in the literature. We call such a model (to be elaborated upon in sequel) a very 
general holographic superconductor (VGHS). The work of [7] dealt with such models in the background of an AdS-Schwarzschild black hole. One of the main purposes of the present paper is to construct such models of holographic superconductors in the background of planar single R-charged black hole solutions including backreaction, and to study features of their HEE. In this introductory section, we will provide a brief overview of the topics to be covered in the rest of the paper and then proceed to summarize our main results.

In the simplest realization of a holographic superconductor, it was shown in [10] that AdS black holes with Abelian Higgs matter become unstable to forming scalar hair near the horizon, below a certain critical temperature $T_{c}$. The main reason for this instability is the presence of a minimal coupling between the scalar and the gauge field, which can make the effective mass term of the scalar field sufficiently negative near the horizon. In the dual boundary field theory, this complex scalar field instability corresponds to a non zero vacuum expectation value $(\mathrm{VEV})$ of the charged scalar operator which is dual to the scalar field in the bulk [2]. The non zero VEV of the scalar operator corresponds to a spontaneous breaking of the $\mathrm{U}(1)$ gauge symmetry and therefore indicates a phase transition from a normal to a superconducting phase, with the scalar operator playing the role of an order parameter. Strictly speaking, at the boundary, it is a global U(1) symmetry which is broken spontaneously and therefore these models more properly describe a holographic charged superfluid. However, one can weakly gauge this symmetry and can still describe superconductivity [4]. Indeed, it was shown explicitly in [2] that the DC conductivity is infinite in these models, which is one of the main characteristic properties of superconductors. Other important features of superconductivity, such as the the existence of an energy gap can also be shown in the context of holographic superconductors [4]. Specifically, in [11], a universal ratio of $\omega_{g} / T_{c} \sim 8$, where $\omega_{g}$ is the gap in the frequency dependent conductivity and $T_{c}$ the critical temperature, was found. A gap in the optical conductivity implies an energy gap in the charge spectrum, which is, as mentioned before, an essential feature of superconductivity. In the weakly coupled BCS theory, $\omega_{g}$ can also be thought as the energy required to break a Cooper pair into its constitutive electrons. Prediction of this ratio from holography, which is twice compared to the BCS theory, indicates the strongly interacting nature of the boundary theory, although by now a large number of exceptions to this result are also known $[12,13]$. Also, Meissner type effects can be shown to exist in holographic superconductors [3, 4].

The original model of holographic superconductors was subsequently generalised in [4] to include the effects of backreaction of the Abelian Higgs matter fields on gravity. Here, it was argued that effects of backreaction do not change the physics too much, and that essentially all the main results are captured by the probe limit. However, there are a few differences and in particular, it was found that the effects of backreaction generally make the condensation harder to form.

An important generalization of the original model of holographic superconductors was considered in [8], where the U(1) symmetry in the boundary is broken by a Stückelberg mechanism. These models have subsequently been called generalized holographic superconductors in the literature. The essential idea here is to consider a non-minimal coupling between the scalar and the gauge field in a gauge invariant way. The importance of this 
models lies in the fact that one can tune the order of the phase transition by introducing additional parameters in the theory, which might be important in realistic systems. With one such parameter, interestingly, several authors found the existence of a first order phase transition from the normal to the superconducting phase, and a metastable region in the superconducting phase $[14,15] .{ }^{1}$ This is phenomenologically important, since there are a number of superconductors which show first order phase transitions $[17,18]$. We should of course emphasize that the first order phase transitions in holographic superconductors are typically studied in the absence of external magnetic fields, unlike real superconductors. However, the issue of of phase transitions in superconductors continue to be an important topic for research in condensed matter physics, and first order transitions inside the superconducting phase at zero magnetic field have seen some interesting development of late [19]. As of now, it is possibly fair to say that predictions from holographic superconductors via AdS/CFT are still far from being tested in the laboratory.

The models mentioned in the previous paragraph are phenomenological in nature, in which the fields and the interactions between them are put in by hand, without actually deriving them from consistent truncations of a string theory, i.e this is a bottom-up approach. In such an approach, the full microscopic discerption of holographic superconductors (like we have for BCS superconductors) are not known. To have such a microscopic description, one has to embed the theory in a string theory, i.e follow a top-down approach (see e.g [20]), which might be substantially more complicated than a bottom-up one, which is the viewpoint we take in this paper. In this bottom-up description, a model with higher derivative interactions of the the scalar and the gauge field via a coupling constant $\eta$ was proposed in [9]. These authors analysed the formation of droplets in an external magnetic field in the probe limit and subsequently also found some signature of the "proximity effect" [21]. A non-trivial generalization of the model of [9] was considered in [7], by introducing two analytical functions of the scalar field in a gauge invariant way. The usefulness of this latter model, which we have called a very general holographic superconductor, lies in the fact that one has multiple tunable parameters in the theory, which provides a far richer phase structure compared to minimally coupled holographic superconductors. For two such parameters, an exotic "window" of first order phase transitions from the normal to the superconducting phase was found in [7]. It is certainly not clear how this might be related to current experimental observations, but if in future, evidence for existence of such systems are found, the VGHS might provide a strong coupling realization of the same.

Now we turn to the concept of entanglement entropy, which has also received a lot of attention of late, and is considered in the later part of this paper. Qualitatively speaking, if a quantum system is divided into two subsystems $\mathcal{A}$ and $\mathcal{B}$, measurements on $\mathcal{A}$ will affect those on $\mathcal{B}$, if the two subsystems are entangled. Entanglement entropy (EE) is a quantitative measure which tells us how strongly these two subsystems are entangled or correlated. Since EE is related to the degrees of freedom of the system, in condensed matter physics it is an important tool to quantify the appearance of a phase transition, as well as its order. However, it is difficult to calculate the EE of a quantum field theory beyond

\footnotetext{
${ }^{1}$ See [16] for a treatment of generalized superconductor with backreaction effects.
} 
$1+1$ dimensions. This problem was bypassed by Ryu and Takayanagi [5], who proposed a simple formula to calculate the EE in the holographic scenario, which is now referred to as the holographic entanglement entropy. Several computations of the HEE have been done using the Ryu-Takayanagi prescription in different contexts, and the results are in good agreement with the standard CFT results.

Recently, [6] used this prescription to study HEE for a "strip geometry" (to be elaborated upon later in this paper) in the context of holographic metal-superconductor phase transitions, and showed that the HEE not only captures the appearance of the phase transition but also its order. The results of this paper also show that for a fixed strip geometry, the HEE in the superconducting phase is always less than that in the normal phase. Naively, this might be thought of as an indicator to the fact that some of the degrees of freedom condense below the transition temperature, but we have to be careful with such a statement. The relationship between the entanglement entropy and the number of the degrees of freedom is subtle. Recently, in the important work of [22], the notion of a "renormalized entanglement entropy," was put forward. For any renormalizable quantum field theory in any dimension, this is defined via the entanglement entropy across a smooth surface characterized by a size, and interpolates between values at the UV and IR fixed points, as the scale size is varied. As shown in [22], for a CFT in any dimension, when the entangling surface is spherical, this is given by the central charge, and thus may be an indicator of the number of the degrees of freedom of the system. In this work, since we compute the HEE using a strip geometry as the entangling surface, we can not obviously relate this to the number of degrees of freedom of the system. ${ }^{2}$ Nevertheless, in this geometry also, the HEE naturally captures the physics of phase transitions as we will show later on in this work.

Subsequent to the work of [6], in [14] and [24], analysis of the behavior of the HEE in the context of holographic insulator-superconductor phase transitions was done. In [21], a holographic superconductor with higher derivative couplings is considered, and these authors calculate the entanglement entropy to study the proximity effect in superconductors. Another recent development in the context of HEE is the interesting notion of the entangling temperature, which first appeared in [25]. In this paper, it was shown that there exists an analogue of the first law of thermodynamics with the HEE playing the role of the usual entropy. For a small subsystem, the change in HEE is proportional to the change in the energy of the subsystem and the proportionality constant, which is given by the size of the entangling region, is interpreted as the inverse of the entangling temperature.

Having briefly reviewed known literature on the topic, we now state our intent. The purpose of the present paper is to extend and complete the study of the VGHS in R-charged backgrounds, in lines with the discussion above. The organization of this paper and the main results contained herein are summarized below.

- In section 2, we construct the VGHS in four dimensional planar R-charged black hole

\footnotetext{
${ }^{2}$ We thank the anonymous referee for pointing this out. It would be interesting to compute the HEE and characterize the phase transition using a spherical geometry as the entangling surface, which might then make its relation to the degrees of freedom clearer. We will however leave this for a future issue.
} 


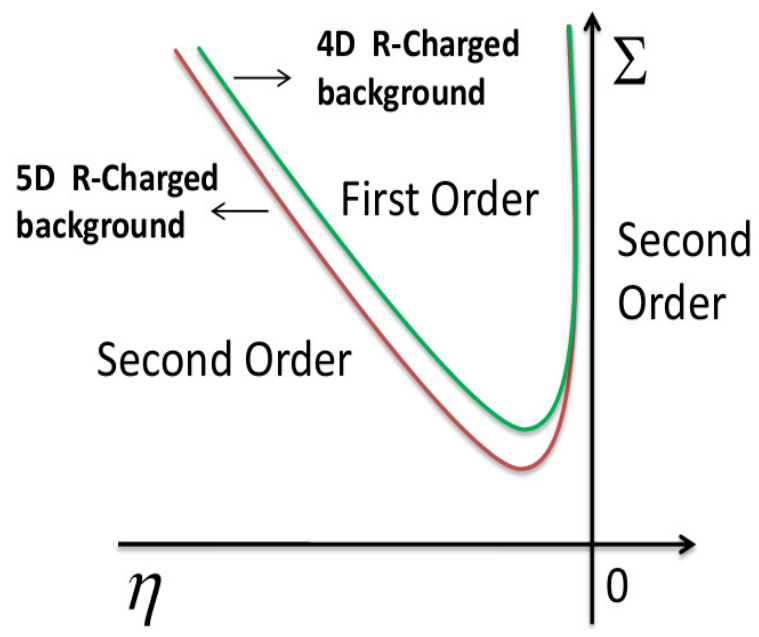

Figure 1. Qualitative phase diagram of the VGHS in the parameter space in the probe limit.

backgrounds, including back reaction effects. We show that in these backgrounds also, there is a window of first order metal - superconductor phase transitions, i.e these first order transitions appear when one appropriately tunes the parameters of the theory in a certain range. We check this result by establishing the nature of the difference in the free energy between the superconductor and the normal phases. This is done for R-charged black hole backgrounds in four and five dimensions. A qualitative phase diagram for our VGHS in the probe limit is shown in figure 1.

- Section 3 is devoted to the study of the VGHS in five dimensional planar R-charged backgrounds. Since the analysis is qualitatively similar to the one carried out in section 2, we relegate the details of calculation in this section to appendix A. Here also we find a window of first order phase transitions within a certain range of parameters of the theory. Again, this is validated by calculating the free energy.

- Next, in section 4, we study holographic entanglement entropy for the R-charged backgrounds studied above. As a warm up exercise, we first calculate the HEE for the VGHS in an AdS Schwarzschild background, and show that the HEE correctly captures the information about the window of first order phase transitions that we have mentioned. (The details of the gravity side of this calculation are relegated to appendix B). We then perform the analysis for R-charged backgrounds and show that the HEE is again an effective tool to pinpoint the window of first order phase transitions in these cases. However, unlike other cases studied in the literature, we find that the HEE for four dimensional R-charged background actually seems to increases in the superconducting phases (compared to the normal phase) whereas the free energy shows expected behavior. Currently, we do not have a proper physical explanation for this, nevertheless, we will provide some discussions towards the end of this section. This feature is absent in five dimensional backgrounds.

- In section 5, we study the entangling temperature for the VGHS, to look for relations 
similar to the first law of thermodynamics with HEE. Our method here is numerical, and we fit the metric and the backreaction parameter with appropriate polynomial functions and extract the entangling temperature. We find some expected variations from the results obtained in [25]. In this section, we confine ourselves to AdSSchwarzschild backgrounds, and point out some difficulties of a similar calculation in R-charged examples. Our results broadly indicate the need to understand better aspects of the entangling temperature for the VGHS in R-charged backgrounds.

- Finally, section 6 ends this paper with a summary of our results and possible directions for future research.

\section{4-D R-charged black hole backgrounds}

In this section we will set up a model for the VGHS in four dimensional R-charged backgrounds. This will also serve to illustrate the basic notations and conventions used in the rest of the paper. We mention in the outset that we will deal with planar R-charged backgrounds with a single charge turned on. With multiple chemical potentials, the solution seems to be intractable.

Recall that R-charged black holes form the gravity duals to rotating branes in various dimensions. As an example, while the gravity dual to a D3-brane configuration is $\operatorname{AdS}_{5} \times S^{5}$, adding spin to the D3-brane configuration in directions orthogonal to its world volume amounts to adding rotations that correspond to a global SO(6) R-symmetry of the $N=4$ conformal field theory that resides on the brane and is related to the $\mathrm{SO}(6)$ symmetry of the $D=5, N=8$ gauged supergravity that arises upon a Kaluza Klein reduction of the spinning brane configuration on $S^{5}$. The three U(1) gauge charges in the $\mathrm{AdS}_{5}$ supergravity are thus related to the spins on the brane world volume, and give rise to three chemical potentials. In a similar manner, black holes in four dimensional $N=8$ AdS supergravity contains four R-charges that correspond to an $\mathrm{SO}(8)$ gauge symmetry arising out of a Kaluza Klein reduction of spinning M2-brane configurations on $S^{7}$. Holographic superconductors can be built by considering an Abelian Higgs model in these geometries.

For the four dimensional single R-charged black hole, we start with the following action

$$
\begin{aligned}
S=\int \mathrm{d}^{4} x \sqrt{-g}[ & \frac{1}{2 \kappa^{2}}\left(R+\frac{3}{L^{2}}\left(H^{1 / 2}+H^{-1 / 2}\right)\right)-\frac{L^{2} H^{3 / 2}}{8} F_{\mu \nu} F^{\mu \nu}-\frac{3}{8} \frac{(\partial H)^{2}}{H^{2}} \\
& \left.-\frac{1}{2}|D \tilde{\Psi}|^{2}-\frac{1}{2} m^{2}|\tilde{\Psi}|^{2}-\frac{\eta}{2}\left|F_{\mu \nu} D^{\nu} \tilde{\Psi}\right|^{2}\right]
\end{aligned}
$$

Here, $\kappa$ is related to the four dimensional Newton's constant, $L$ is the AdS length scale and $\tilde{\Psi}$ is a complex scalar field with charge $q$ and mass $m$. Also, $F=d A$ and $D_{\mu}=\partial_{\mu}-i q A_{\mu}$. For $\tilde{\Psi}=0$, the above action reduces to that of the single R-charged black hole background (see e.g [28]) with $H(r)=1+k r_{h} / r, r_{h}$ being the horizon radius, and $k$ a charge parameter. Also, the last term in eq. (2.1) describes the higher derivative interaction between the scalar field and the field strength tensor. The form of the interaction can be motivated from a Landau-Ginzburg analysis, but we will prefer to study this from a phenomenological point 
of view. Rewriting the charged scalar field $\tilde{\Psi}=\Psi e^{i \alpha}$, the action can be cast as

$$
\begin{aligned}
S=\int \mathrm{d}^{4} x \sqrt{-g}[ & \frac{1}{2 \kappa^{2}}\left(R+\frac{3}{L^{2}}\left(H^{1 / 2}+H^{-1 / 2}\right)\right)-\frac{L^{2} H^{3 / 2}}{8} F_{\mu \nu} F^{\mu \nu}-\frac{3}{8} \frac{(\partial H)^{2}}{H^{2}} \\
& -\frac{\left(\partial_{\mu} \Psi\right)^{2}}{2}-\frac{m^{2} \Psi^{2}}{2}-\frac{\eta}{2} F_{\mu \nu} \partial^{\nu} \Psi F^{\mu \sigma} \partial_{\sigma} \Psi-\frac{\Psi^{2}(\partial \alpha-q A)^{2}}{2} \\
& \left.-\frac{\eta}{2} \Psi^{2}\left(F^{\mu \nu}\left(\partial_{\nu} \alpha-q A_{\nu}\right)\right)^{2}\right]
\end{aligned}
$$

The $\mathrm{U}(1)$ symmetry in the above action is now given by $\alpha \rightarrow \alpha+q \lambda$ and $A_{\mu} \rightarrow A_{\mu}+\partial_{\mu} \lambda$. Following $[7,8]$, the above action can be generalized in gauge invariant way

$$
\begin{aligned}
S=\int \mathrm{d}^{4} x \sqrt{-g}[ & \frac{1}{2 \kappa^{2}}\left(R+\frac{3}{L^{2}}\left(H^{1 / 2}+H^{-1 / 2}\right)\right)-\frac{L^{2} H^{3 / 2}}{8} F_{\mu \nu} F^{\mu \nu}-\frac{3}{8} \frac{(\partial H)^{2}}{H^{2}} \\
& -\frac{\left(\partial_{\mu} \Psi\right)^{2}}{2}-\frac{\eta}{2} F_{\mu \nu} \partial^{\nu} \Psi F^{\mu \sigma} \partial_{\sigma} \Psi-\frac{m^{2} \Psi^{2}}{2}-\frac{|\mathrm{G}(\Psi)|(\partial \alpha-q A)^{2}}{2} \\
& \left.-\frac{\eta}{2}|\mathrm{~K}(\Psi)|\left(F^{\mu \nu}\left(\partial_{\nu} \alpha-q A_{\nu}\right)\right)^{2}\right]
\end{aligned}
$$

here $\mathrm{G}(\Psi)$ and $\mathrm{K}(\Psi)$ are two analytic functions of $\Psi$ whose general form will be specified in subsequent text. Eq. (2.3) defines the VGHS. If $\mathrm{K}(\Psi)=\Psi^{2}$, we will obtain the generalized holographic superconductor of [8] along with a higher derivative coupling. However, as we show in sequel, more general forms of $\mathrm{K}(\Psi)$ leads to a rich phase structure in the theory. ${ }^{3}$

Now for hairy black hole like solutions with backreaction, we consider the following ansatz $^{4}$

$$
\begin{aligned}
d s^{2} & =-g(r) H(r)^{-1 / 2} e^{-\chi(r)} d t^{2}+H(r)^{1 / 2} r^{2}\left(d x^{2}+d y^{2}\right)+H(r)^{1 / 2} \frac{d r^{2}}{g(r)} \\
\Psi & =\Psi(r), \quad A=\Phi(r) d t
\end{aligned}
$$

We will henceforth consider a particular gauge where $\alpha=0$. In this gauge, the equation of motion for the scalar field $\Psi$ can be obtained as

$$
\begin{aligned}
\Psi^{\prime \prime}\left(1-\eta e^{\chi} \Phi^{\prime 2}\right) & +\frac{H e^{\chi} \Phi^{2}}{2 g^{2}} \frac{d \mathrm{G}(\Psi)}{d \Psi}-\frac{\eta H e^{2 \chi} \Phi^{2} \Phi^{\prime 2}}{2 g^{2}} \frac{d \mathrm{~K}(\Psi)}{d \Psi}+\Psi^{\prime}\left(\frac{2}{r}+\frac{g^{\prime}}{g}-\frac{\chi^{\prime}}{2}\right) \\
& -\frac{m^{2} H^{1 / 2} \Psi}{g}-\eta \Psi^{\prime}\left(\frac{e^{\chi} g^{\prime} \Phi^{\prime 2}}{g}+\frac{e^{\chi} \Phi^{\prime 2} \chi^{\prime}}{2}+\frac{2 e^{\chi} \Phi^{\prime 2}}{r}+2 e^{\chi} \Phi^{\prime} \Phi^{\prime \prime}\right)=0
\end{aligned}
$$

Similarly, we get the equation of motion for the zeroth component of the gauge field as

$$
\begin{aligned}
& \Phi^{\prime \prime}\left(1-\frac{2 \eta e^{\chi} \Phi^{2} \mathrm{~K}(\Psi)}{g H}+\frac{2 \eta g \Psi^{\prime 2}}{H^{2}}\right)-\Phi\left(\frac{2 \mathrm{G}(\Psi)}{g H}+\frac{2 \eta e^{\chi} \Phi^{\prime 2} \mathrm{~K}(\Psi)}{g H}\right) \\
& +\Phi^{\prime}\left(\frac{2 \eta g^{\prime} \Psi^{\prime 2}}{H^{2}}+\frac{\eta g \chi^{\prime} \Psi^{\prime 2}}{H^{2}}+\frac{4 \eta g \Psi^{\prime 2}}{r H^{2}}+\frac{4 \eta g \Psi^{\prime} \Psi^{\prime \prime}}{H^{2}}+\frac{2}{r}+\frac{2 H^{\prime}}{H}+\frac{\chi^{\prime}}{2}\right) \\
& +2 \eta \mathrm{K}(\Psi) \Phi^{2} \Phi^{\prime}\left(\frac{e^{\chi} g^{\prime}}{g^{2} H}-\frac{e^{\chi} \mathrm{K}(\Psi)^{\prime}}{g H \mathrm{~K}(\Psi)}-\frac{3 e^{\chi} \chi^{\prime}}{2 g H}-\frac{2 e^{\chi}}{r g H}-\frac{e^{\chi} H^{\prime}}{g H^{2}}\right)=0
\end{aligned}
$$

\footnotetext{
${ }^{3}$ In the rest of this paper, we perform the computations by setting $L=1$ and $q=1$.

${ }^{4}$ All numerical calculations in this paper are performed using MATHEMATICA routines. We find that in some situations, a conformally equivalent metric ansatz $d s^{2}=-g(r) e^{-\xi(r)} d t^{2}+r^{2}\left(d x^{2}+d y^{2}\right)+\frac{d r^{2}}{g(r)}$ reduces the computation time considerably, while giving the same numerical results as when one uses eq. (2.4).
} 
Also, the equation of motion for the $H$ field is given by

$$
H^{\prime \prime}+H^{\prime}\left(\frac{2}{r}+\frac{g^{\prime}}{g}-\frac{\chi^{\prime}}{2}-\frac{H^{\prime}}{H}\right)+\frac{e^{\chi} H^{3} \Phi^{\prime 2}}{2 g}+\frac{2 H}{2 \kappa^{2} g}(H-1)=0
$$

Finally, the $r r$ and the $(t t-r r)$ components of Einstein equation give

$$
\begin{gathered}
g^{\prime}-g \chi^{\prime}+\frac{g}{r}-\frac{3 r}{2}(H+1)+\frac{r g H^{\prime}}{4 H}\left(\frac{g^{\prime}}{g}-\frac{H^{\prime}}{4 H}-\chi^{\prime}\right) \\
+2 \kappa^{2} r\left(-\frac{H e^{\chi} \Phi^{2} \mathrm{G}(\Psi)}{4 g}+\frac{H^{1 / 2} m^{2} \Psi^{2}}{4}-\frac{3 g H^{\prime 2}}{16 H^{2}}+\frac{3 \eta g e^{\chi} \Phi^{\prime 2} \Psi^{\prime 2}}{4}\right. \\
\left.+\frac{e^{\chi} H^{2} \Phi^{\prime 2}}{8}-\frac{\eta H e^{2 \chi} \Phi^{2} \Phi^{\prime 2} \mathrm{~K}(\Psi)}{4 g}-\frac{g \Psi^{\prime 2}}{4}\right)=0 \\
2 \kappa^{2} r\left(\frac{H e^{\chi} \Phi^{2} \mathrm{G}(\Psi)}{2 g^{2}}+\frac{3 H^{\prime 2}}{8 H^{2}}-\frac{\eta H e^{2 \chi} \Phi^{2} \Phi^{\prime 2} \mathrm{~K}(\Psi)}{2 g^{2}}+\frac{\Psi^{\prime 2}}{2}-\frac{\eta e^{\chi} \Phi^{\prime 2} \Psi^{\prime 2}}{2}\right) \\
\chi^{\prime}+\frac{H^{\prime}}{H}-\frac{3 r H^{\prime} 2}{8 H^{2}}+\frac{r H^{\prime} \chi^{\prime}}{4 H}+\frac{r H^{\prime \prime}}{2 H}=0
\end{gathered}
$$

In the above equations, we have explicitly suppressed the radial dependence of our variables, and the prime denotes a derivative with respect to the radial coordinate $r$. Let us record the expression for the Hawking temperature of the black hole with the geometry in equation (2.4), which is given by

$$
T_{H}=\left.\frac{g^{\prime}(r) e^{-\chi(r) / 2}}{4 \pi \sqrt{H(r)}}\right|_{r=r_{h}}
$$

where $r_{h}$, the radius of the event horizon, is given by the solution of $g\left(r_{h}\right)=0$. Finally therefore, we have five coupled differential equations which need to be solved with appropriate boundary conditions. We impose the regularity conditions for $\Phi$ and $\Psi$ at the horizon

$$
\Phi\left(r_{h}\right)=0, \quad \Psi^{\prime}\left(r_{h}\right)=\frac{m^{2} \sqrt{H\left(r_{h}\right)} \Psi\left(r_{h}\right)}{g^{\prime}\left(r_{h}\right)\left(1-\eta e^{\chi\left(r_{h}\right)} \Phi^{\prime 2}\left(r_{h}\right)\right)} .
$$

Near the boundary these fields asymptote to the following expressions

$$
\Phi=\mu-\frac{\rho}{r}+\ldots, \quad \Psi=\frac{\Psi_{-}}{r^{\lambda_{-}}}+\frac{\Psi_{+}}{r^{\lambda_{+}}}+\ldots \quad \chi \rightarrow 0, \quad g \rightarrow r^{2}+\ldots, \quad H \rightarrow 1+\ldots
$$

where $\mu$ and $\rho$ are interpreted as the the chemical potential and the charge density of the boundary theory respectively, and $\lambda_{ \pm}=\frac{3 \pm \sqrt{9+4 m^{2}}}{2}$. In this paper we consider a special case with $m^{2}=-2$ which also implies $\lambda_{ \pm}=2,1$. Although $m^{2}$ is negative but it is above the Breitenlohner-Freedman (BF) bound $m^{2}=-9 / 4$ in four spacetime dimensions. Now some interpretation of the boundary parameters in eq. (2.13) are in order. We will interpret the leading falloff $\Psi_{-}$as the source term and the subleading term $\Psi_{+} \sim \mathrm{O}_{2}$ as the VEV of the dual scalar operator. With $m^{2}=-2$, the meaning of $\Psi_{-}$and $\Psi_{+}$can also be interchangeable though this scenario is not considered in this paper. Since we want 


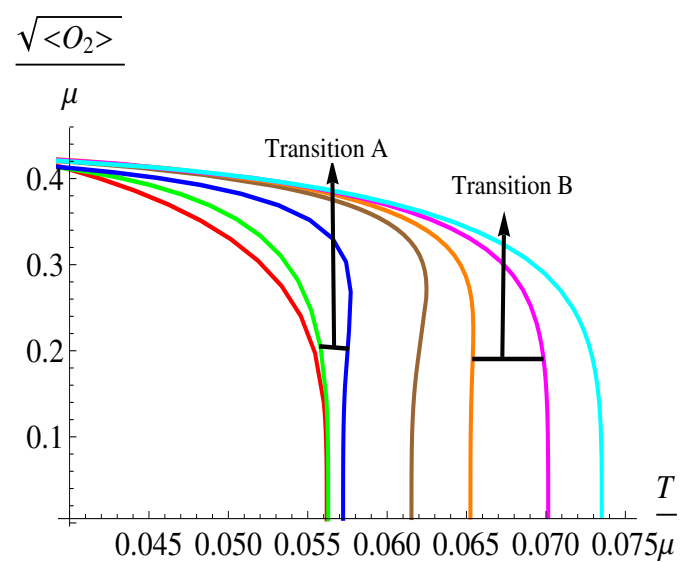

Figure 2. Variation of the condensate for different values of $\eta$ with fixed $\Sigma=10$ and $2 \kappa^{2}=0.3$ for $4 \mathrm{D}$ R-charged black hole background.

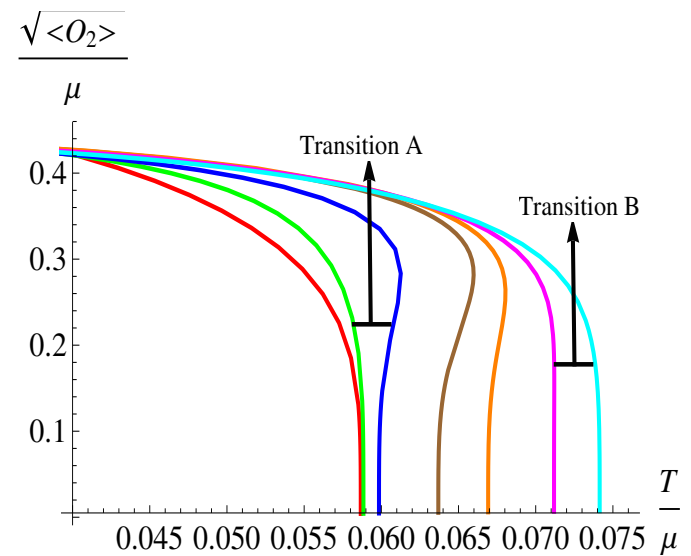

Figure 4. Variation of the condensate for different values of $\eta$ with fixed $\Sigma=10$ and $2 \kappa^{2}=0$ for $4 \mathrm{D}$ R-charged black hole background.

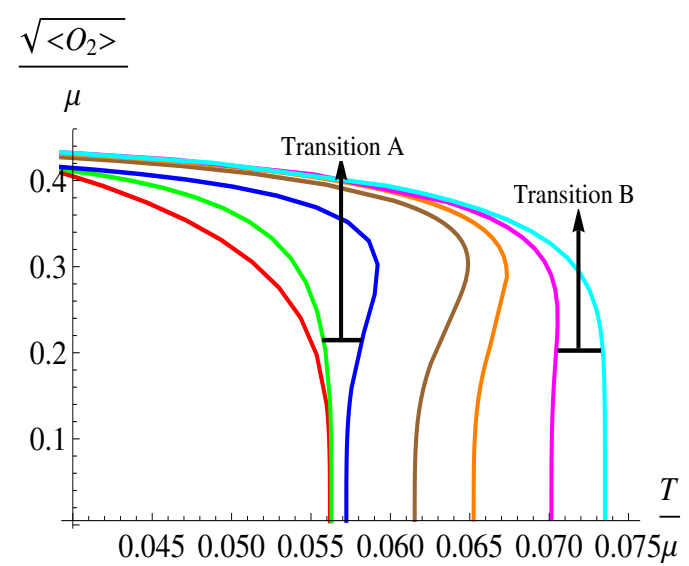

Figure 3. Variation of the condensate for different values of $\eta$ with fixed $\Sigma=15$ and $2 \kappa^{2}=0.3$ for $4 \mathrm{D}$ R-charged black hole background.

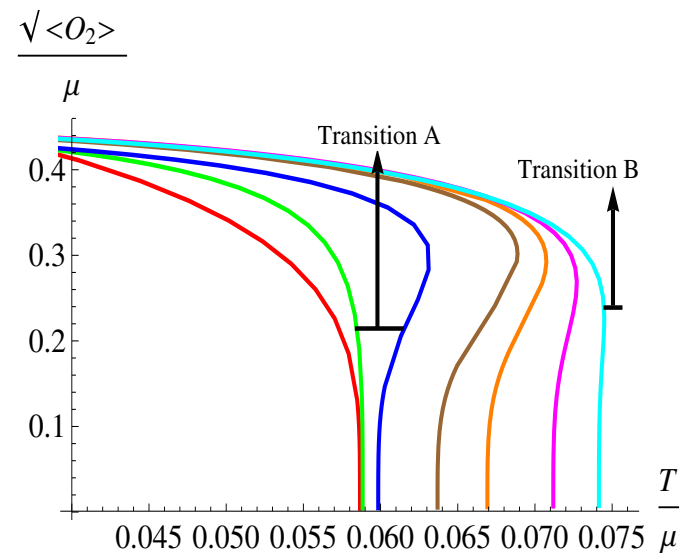

Figure 5. Variation of the condensate for different values of $\eta$ with fixed $\Sigma=15$ and $2 \kappa^{2}=0$ for $4 \mathrm{D}$ R-charged black hole background.

to break the U(1) symmetry spontaneously, we will set the source term $\Psi_{-}=0$ as the boundary condition.

In the equations of motion (2.6)-(2.10), we will consider particular forms of $G(\Psi)$ and $\mathrm{K}(\Psi)$ :

$$
\mathrm{G}(\Psi)=\Psi^{2}+\xi \Psi^{\theta}, \quad \mathrm{K}(\Psi)=\Psi^{2}+\Sigma \Psi^{\gamma}
$$

As in [7], we are mostly interested in examining the phase structure of the boundary superconductors with respect to $\eta$ and $\Sigma$. For this reason we will set the other parameters to a fixed value, in particular $\xi=0$ and $\gamma=4$, but we have checked for several examples that a non zero value of $\xi$ and different values of $\gamma$ do not change the results qualitatively.

Now we present numerical results on the VGHS. ${ }^{5}$ In figures 2 and 3, we show the plots of the condensate $\sqrt{\left\langle\mathrm{O}_{2}\right\rangle}$ with a back reaction parameter $2 \kappa^{2}=0.3$, for various values of

\footnotetext{
${ }^{5}$ For numerical convenience, we use the $z=r_{h} / r$ coordinate.
} 
$\eta$, with $\Sigma=10$ and 15 respectively. In these figures, the red, green, blue, brown, orange, magenta and cyan curves corresponds to $\eta=0.01,-0.01,-0.1,-0.5,-1,-2$ and -3 , respectively. One can notice the non zero value of the condensate below a certain critical $T / \mu$ which indicates the onset of the superconducting phase. Above this $T / \mu$, the system is in the normal phase where the condensate is zero. We see from figures 2 and 3 that there is an interesting window of first order phase transitions: as we decrease the higher derivative coupling parameter $\eta$, the transition - which was of second order for positive values of $\eta$ - changes order, and it remains first order within a range of $\eta$. This range appears to increase with an increase of the value of $\Sigma$. This is qualitatively indicated in figures 2 and 3, where "transition A" refers to the order of the phase transition changing from second to first, and the reverse for "transition B."

In figures 4 and 5 , we have also shown the condensate calculations for $2 \kappa^{2}=0$, which corresponds to the probe limit in our model. Analysis of these results indicate that the backreaction parameter for the VGHS in R-charged black hole backgrounds makes the window in $\eta$, with in which the transition from normal to superconducting phase is first order, narrower compared to the probe limit. This is in stark contrast with the results obtained with the AdS-Schwarzschild black hole background, where increase in backreaction parameter makes the window in $\eta$ wider compared to the probe limit [7]. This is a non-trivial effect of the spin of the brane configuration.

In the same spirit, figures 6 and 7 show the condensate values for the VGHS, for the same back reaction parameter, for various values of $\Sigma$, with $\eta$ fixed at -0.1 and -3 respectively. For both these graphs, the red, green, blue, brown and orange curves corresponds to $\Sigma=1,5,7,10$ and 15 , respectively. We find that for a fixed value of $\eta$, the transition from the normal to the superconductor phase does not have a window (where the transition is of first order), contrary to the case of fixed $\Sigma$. In the present case, for small negative values of $\eta$, the order of the transition changes from second to first, as one increases $\Sigma$. This suggest the existence of a lower cutoff in $\Sigma\left(\Sigma_{c}\right)$ above which the phase transition from the normal to the superconducting phase is of first order. For further lower values of $\eta$, in the range of $\Sigma$ considered here, the normal to superconductor transition is always of second order. Qualitatively, this was the behavior alluded to in the introduction, in figure 1. For the sake of comparison with the VGHS in AdS-Schwarzschild black hole backgrounds, we also note that the value of the cutoff $\Sigma_{c}$ is larger for our R-charged background.

A word about the magnitude of the critical $T / \mu$ is in order. Normally, higher backreaction parameter makes the critical $T / \mu$ smaller, which generally implies that the backreaction makes the scalar condensation harder to form. This is also the case here. In a similar manner, the critical value of $T / \mu$ also decreases for higher values of $\eta$ but, on the other hand, does not depends on $\Sigma$. This is expected from a physical ground in eq. (2.14), since at the phase transition point $\Psi$ is negligible and therefore $\Sigma$ which comes with higher powers of $\Psi$ does not have any effect on critical $T / \mu$. We mention here the overall behavior of critical $T / \mu$ in R-charged black hole backgrounds for different value of $\kappa$ and $\eta$, is the same as in AdS-Schwarzschild black hole background but with higher magnitude. This indicates that the scalar field instability is easier to form in a VGHS for spinning brane configurations. 


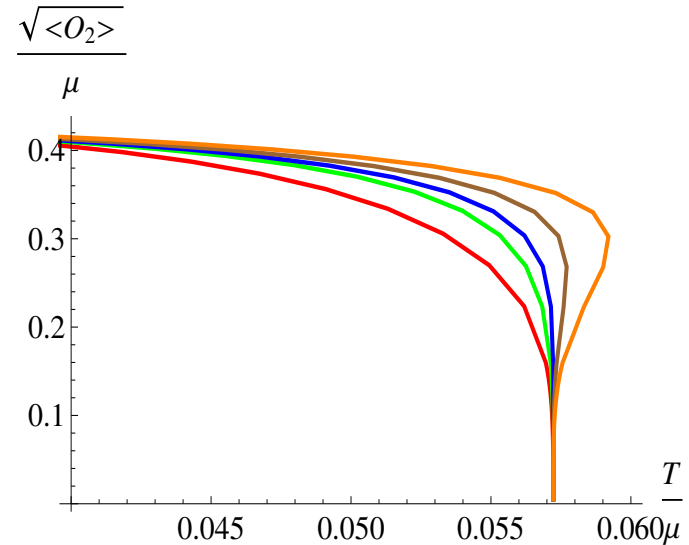

Figure 6. Variation of condensate for different values of $\Sigma$ with fixed $\eta=-0.1$ and $2 \kappa^{2}=0.3$ for $4 \mathrm{D}$ R-charged black hole background.

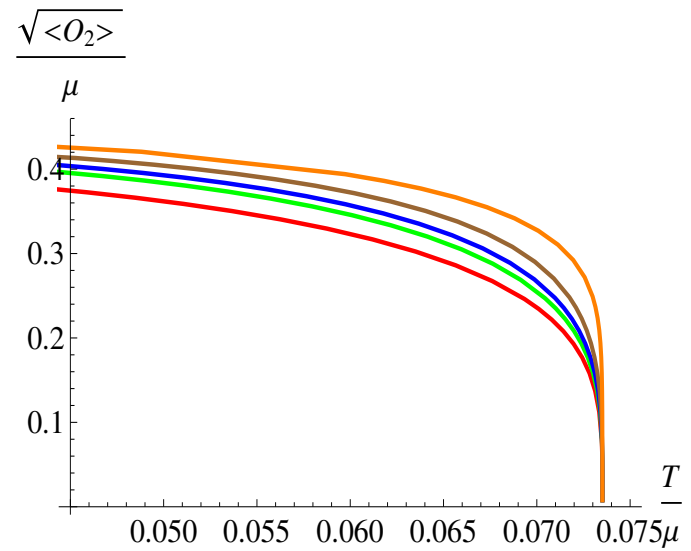

Figure 7. Variation of condensate for different values of $\Sigma$ with fixed $\eta=-3$ and $2 \kappa^{2}=0.3$ for $4 \mathrm{D}$ R-charged black hole background.

To check the validity of this result, it is worthwhile to understand the behavior of the free energy in these cases. To highlight the essential physics, it is enough to consider the probe limit, with $\kappa^{2}=0$ and compute the Gibbs free energy of the boundary thermal state by identifying the latter with the bulk on-shell action. As usual, one has to add a boundary counter term to the on-shell action, and calculate the renormalized free energy. Following this procedure, we find that the difference of free energy between the normal and the superconducting phase is given by the expression

$$
\begin{aligned}
\Delta \Omega= & \Omega_{\text {Superconductor }}-\Omega_{\text {Normal }} \\
= & -\frac{\mu \rho}{4}+\frac{1}{2} \int_{0}^{1} \mathrm{~d} z \frac{\Phi(z)^{2} \Psi(z)^{2}}{z^{4} g(z)}+\frac{\eta}{2} \int_{0}^{1} \mathrm{~d} z z^{4} g(z) \Phi^{\prime}(z)^{2} \Psi^{\prime}(z)^{2} \\
& -\frac{\eta}{2} \int_{0}^{1} \mathrm{~d} z \frac{\Phi(z)^{2} \Phi^{\prime}(z)^{2} \Psi(z)^{2}}{g(z)}\left(2+3 \Sigma \Psi(z)^{2}\right)+\frac{\mu^{2}}{4}
\end{aligned}
$$

This difference of the free energy is plotted in figures 8 and 9 which use the same colour coding as figure 2 and figure 7 , respectively. The result is exactly as we expect. For fixed $\Sigma$, we find a window of first order phase transitions, which is absent in the analysis with fixed $\eta$. A similar result was obtained in [7] for VGHS in the AdS-Schwarzschild background.

We now proceed to calculate the optical conductivity of our boundary superconducting system. We work with vector type perturbations in the metric and in the gauge field, with $g_{x t} \neq 0, g_{x y} \neq 0$ and $A_{x} \neq 0$. The computation is standard: we assume the spatial and time dependence of the perturbations to be of the form $e^{i k y-i \omega t}$, and work at the linearized level. In this perturbation there are four independent equations. However in the limit $k \rightarrow 0$, which is appropriate to compute the optical conductivity, two of these independent equations - namely the $x t$ and the $x y$ components of the Einstein equations - decouple. After rearranging the other two equations, we find a second order differential equation for 

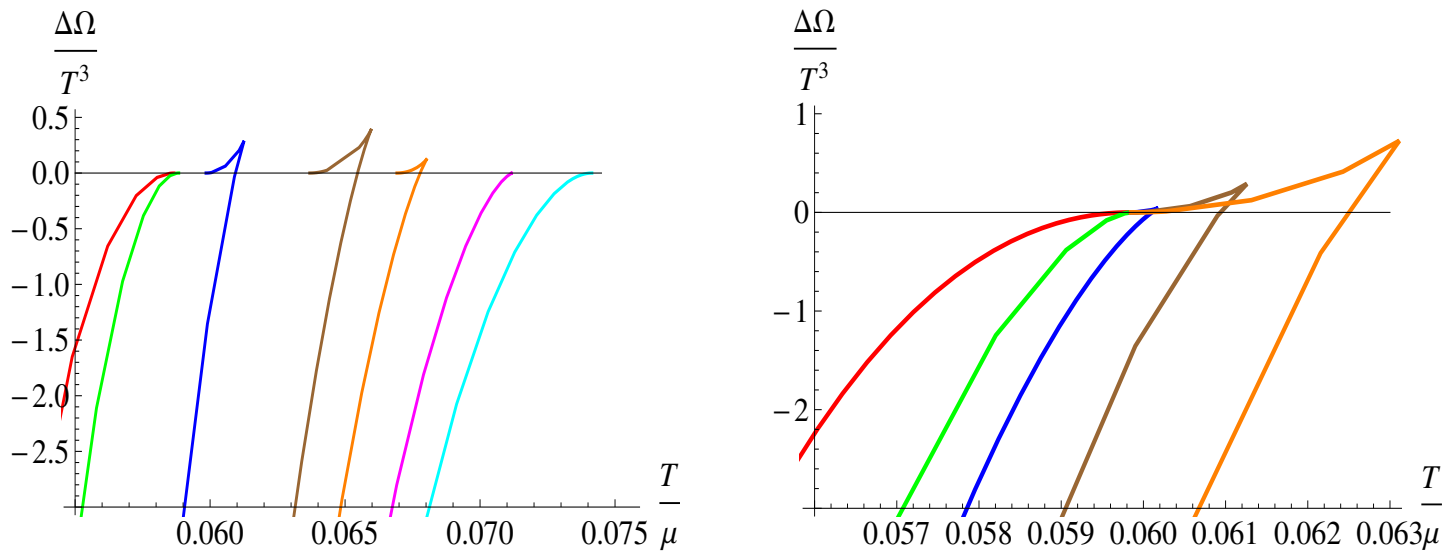

Figure 8. Difference in free energy between Figure 9. Difference in free energy between the superconducting and normal phase in $4 \mathrm{D} R$ the superconducting and normal phase in $4 \mathrm{D}$ charged background for fixed $\Sigma=10$ and $2 \kappa^{2}=0 \quad \mathrm{R}$ charged background for fixed $\eta=-0.1$ and for different values of $\eta$. Here we have used the $2 \kappa^{2}=0$ for different values of $\Sigma$. Here we have same color coding as in figure 2 . used the same color coding as in figure 6 .
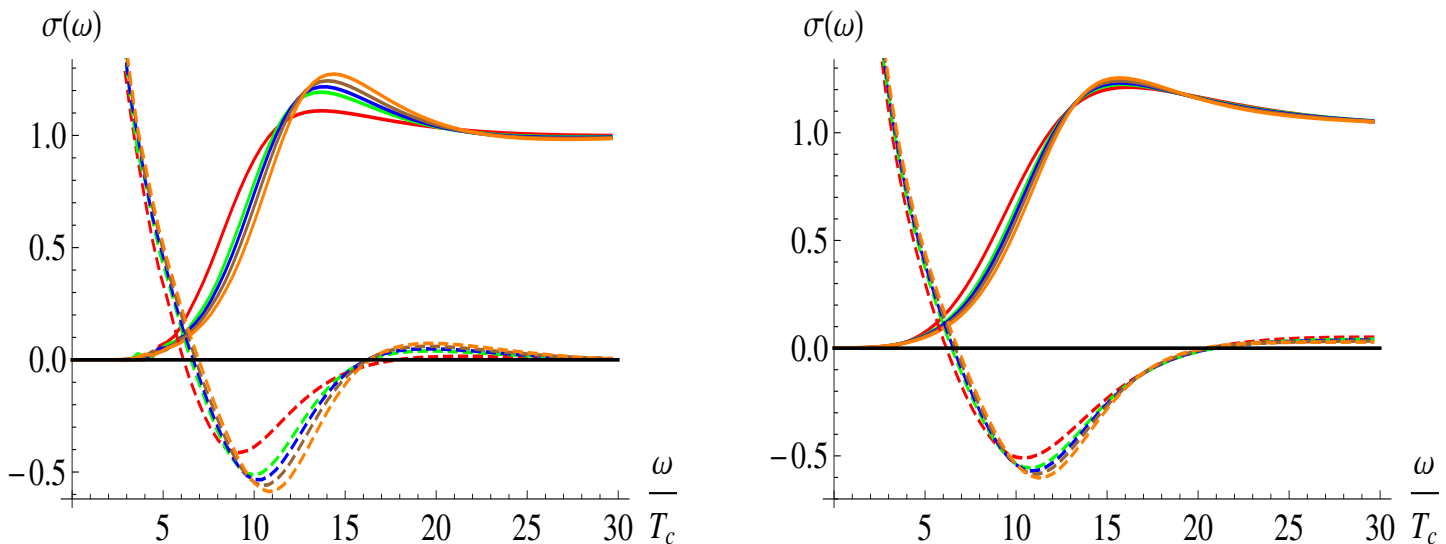

Figure 10. Real (solid lines) and imaginary Figure 11. Real (solid lines) and imaginary (dotted lines) part of conductivity for different (dotted lines) part of conductivity for different values of $\Sigma$ with fixed $\eta=-0.1$ and $2 \kappa^{2}=0$ for values of $\Sigma$ with fixed $\eta=-0.1$ and $2 \kappa^{2}=0.3$ $4 \mathrm{D}$ R-charged black hole background.

for 4D R-charged black hole background.

$A_{x}$, which is given by

$$
\begin{aligned}
A_{x}^{\prime \prime}\left(1+\frac{2 \eta g \psi^{\prime 2}}{H^{2}}\right)+A_{x}^{\prime}\left(\frac{g^{\prime}}{g}+\frac{H^{\prime}}{H}-\frac{\chi^{\prime}}{2}\right)+\frac{\eta \psi^{\prime 2} A_{x}^{\prime}}{H^{2}}\left(4 g^{\prime}-g \chi^{\prime}+\frac{4 g \psi^{\prime \prime}}{\psi^{\prime}}-\frac{2 g H^{\prime}}{H}\right) \\
+2 \kappa^{2} \eta^{2} e^{\chi} A_{x}\left(-\frac{2 e^{2 \chi} \phi^{4} \phi^{\prime 2} \mathrm{~K}(\Psi)^{2}}{g^{3}}+\frac{4 e^{\chi} \phi^{2} \phi^{\prime 2} \psi^{\prime 2} \mathrm{~K}(\Psi)}{g H}-\frac{2 g \phi^{\prime 2} \psi^{\prime 4}}{H^{2}}\right) \\
+A_{x}\left(\frac{e^{\chi} H \omega^{2}}{g^{2}}-\frac{2 \mathrm{G}(\Psi)}{g H}-\frac{2 \kappa^{2} e^{\chi} H^{2} \phi^{\prime 2}}{2 g}\right)+\eta e^{\chi} \mathrm{K}(\Psi) A_{x}\left(-\frac{2 e^{\chi} \omega^{2} \phi^{2}}{g^{3}}\right. \\
\left.-\frac{2 \phi \phi^{\prime} \mathrm{K}(\Psi)^{\prime}}{g H \mathrm{~K}(\Psi)}-\frac{2 \phi^{\prime 2}}{g H}+\frac{4 \kappa^{2} e^{\chi} H \phi^{2} \phi^{\prime 2}}{g^{2}}-\frac{\phi \phi^{\prime} \chi^{\prime}}{g H}-\frac{4 \kappa^{2} \phi^{\prime 2} \psi^{\prime 2}}{\mathrm{~K}(\Psi)}-\frac{2 \phi \phi^{\prime \prime}}{g H}\right)=0
\end{aligned}
$$


where we have again suppressed the $r$-dependence. In order to solve this equation we need to apply appropriate boundary conditions. At the horizon, we impose an infalling boundary condition $A_{x} \alpha g(r)^{-i \omega / 4 \pi T_{h}}$. At the asymptotic boundary, $A_{x}$ behaves as

$$
A_{x}=A_{x}^{(0)}+\frac{A_{x}^{(1)}}{r}+\ldots
$$

Using the AdS/CFT prescription, one can identify the leading term $A_{x}^{(0)}$ and the subleading term $A_{x}^{(1)}$ as the dual source and the expectation value of boundary current, respectively, and the expression for the conductivity by calculating current-current correlator is given by $[26]$

$$
\sigma(\omega)=-\frac{i A_{x}^{(1)}}{\omega A_{x}^{(0)}}
$$

The results are shown in figures 10 and 11, where we have fixed $\eta=-0.1$ and the red, green, blue, brown and orange curves correspond to $\Sigma=1,5,7,10$ and 15, respectively. While figure 10 corresponds to the probe limit $\kappa^{2}=0$, figure 11 is for a non-zero value of the back reaction, $2 \kappa^{2}=0.3 .{ }^{6}$ From these figures, we can see the signature of the pole in the imaginary part of the conductivity at $\omega=0$. It implies that, using Kramers-Kronig relations which relate the real and imaginary part of the conductivity, the real part of the conductivity has a delta function at $\omega=0$. However, this delta function is not visible in the numerical calculations in figure 10 and 11 due to its infinitesimal width. Another important observation from these figures is the magnitude of gap frequency to the critical temperature, $\omega_{g} / T_{c} \sim 10$, where $\omega_{g}$ is defined as the frequency at which the imaginary part of the conductivity is minimum. Interestingly, this ratio is relatively small compared to the VGHS in AdS-Schwarzschild black hole background where $\omega_{g} / T_{c}$ was found to be nearly 20 [7]. This indicates that the boundary superconductor in an AdS-Schwarzschild black hole background is more strongly coupled than its R-charged cousin. For different value of $\eta$, the results for the conductivity are qualitatively similar.

\section{5-D R-charged black hole backgrounds}

For 5-D R-charged backgrounds, the procedure for constructing a VGHS is entirely similar to what has been discussed in the previous section. We will relegate the details of the computation here to appendix A, and simply present numerical results. Here we have considered $m^{2}=-15 / 4$, which is again above the Breitenlohner-Freedman bound $m_{B F}^{2}=$ -4 for the five dimensional AdS background. ${ }^{7}$

In figure 12, we have plotted the condensate as a function of the temperature for 5D R-charge backgrounds with a fixed values of $\Sigma=10$ and the back reaction parameter $2 \kappa^{2}=0.3$, for different values of the higher derivative coupling parameter $\eta$. Here, the red, green, blue, brown, orange, magenta and cyan curves corresponds to $\eta=0.01,-0.01$, $-0.1,-0.5,-1,-2$ and -3 , respectively. We again get a phase transition from normal

\footnotetext{
${ }^{6}$ Here the temperature is measured in units of $\rho$ and we have chosen $T=0.2 T_{c}$.

${ }^{7}$ For the interpretation of various physical quantities, see appendix A.
} 


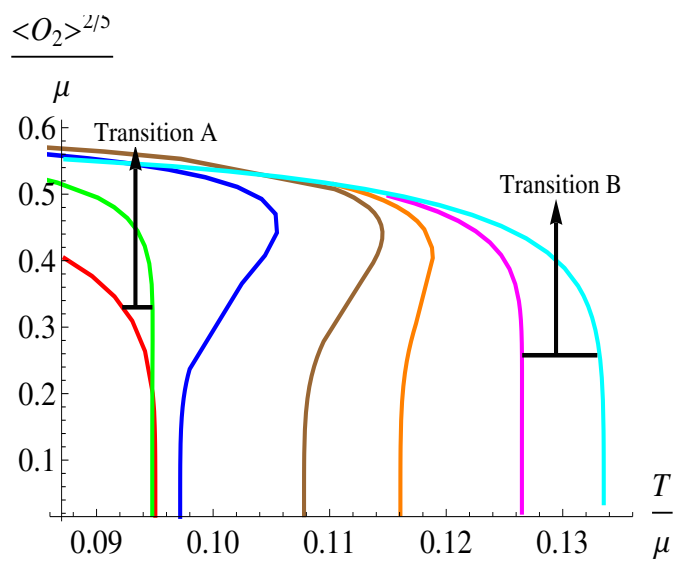

Figure 12. Variation of the condensate for different values of $\eta$ with fixed $\Sigma=10$ and $2 \kappa^{2}=0.3$ for 5D R-charged black hole background.

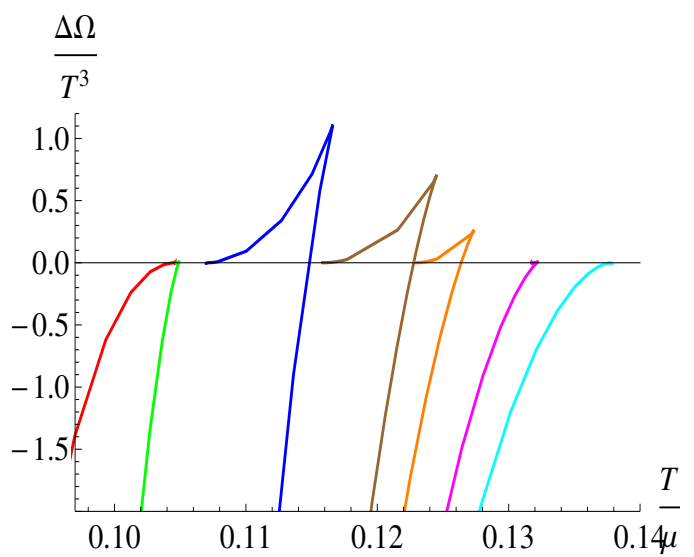

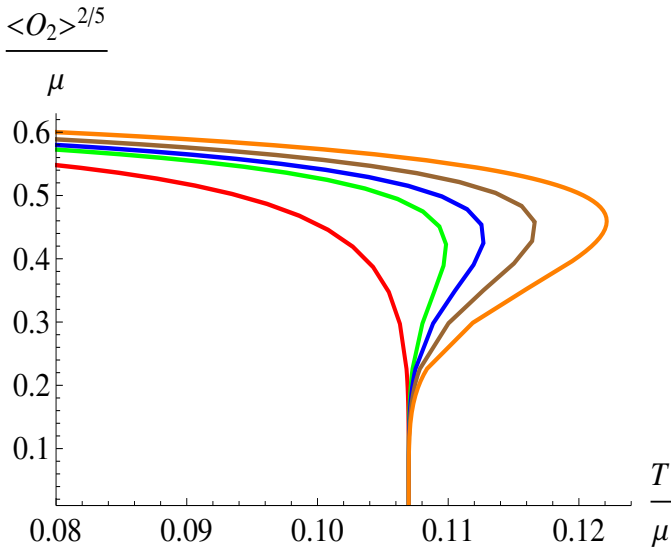

Figure 13. Variation of the condensate for different values of $\Sigma$ with fixed $\eta=-0.1$ and $2 \kappa^{2}=0$ for $5 \mathrm{D}$ R-charged black hole background.

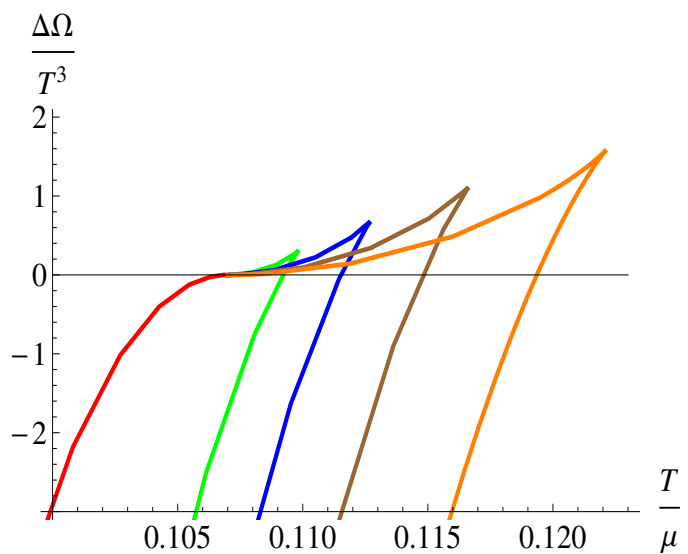

Figure 14. Difference in free energy between Figure 15. Difference in free energy between the superconducting and normal phase in 5-D R the superconducting and normal phase in 5-D R charged background for fixed $\Sigma=10$ and $2 \kappa^{2}=0$ charged background for fixed $\eta=-0.1$ and $2 \kappa^{2}=$ for different values of $\eta$.

0 for different values of $\Sigma$.

to superconducting phase below a critical $T / \mu$ and find a window in $\eta$ for the first order phase transitions. However, this window is relatively larger compared to the $4 \mathrm{D}$ R-charged case (figure 2). The same scenario (not presented here) is observed in the probe limit also.

In the probe limit, the condensate is plotted for different values of $\Sigma$ in figure 13, where the red, green, blue, brown and orange curves correspond to $\Sigma=1,5,7,10$ and 15, respectively. We see that the results are qualitatively similar to our computation in the 4-D background with a lower cutoff parameter $\Sigma_{c}$, above which the transition is always of first order.

These results were checked with the corresponding free energy calculations, which are presented in figures 14 and 15. In both these figures, we have chosen the back reaction to be zero, for illustration. In the former case, we get a window of first order phase transitions which is absent in the latter thereby justifying the results we obtained by analysing the condensate. 


\section{HEE for very general holographic superconductors}

In this section, we will compute the holographic entanglement entropy for very general holographic superconductors. For the sake of completeness, we will first recapitulate a few known facts. As mentioned in the introduction, entanglement entropy is a measure of the correlation between two subsystems $\mathcal{A}$ and $\mathcal{B}$ of a given quantum system. Specifically, the entanglement entropy of subsystem $\mathcal{A}$ is given by,

$$
S_{\mathcal{A}}=-\operatorname{Tr}_{\mathcal{A}}\left(\rho_{\mathcal{A}} \ln \rho_{\mathcal{A}}\right) .
$$

where $\rho_{\mathcal{A}}$ is the reduced density matrix of $\mathcal{A}$, calculated by taking the trace over the degrees of freedom of $\mathcal{B}$, i.e, $\rho_{\mathcal{A}}=\operatorname{Tr}_{\mathcal{B}}(\rho), \rho$ being the density matrix of the full quantum system. In a holographic setup, the Ryu-Takayanagi proposal states that the HEE of the subsystem $\mathcal{A}$ living on the boundary of a $(d+1)$ dimensional AdS space is given by,

$$
S_{\mathcal{A}}=\frac{\operatorname{Area}\left(\gamma_{\mathcal{A}}\right)}{4 G_{N}}
$$

where $G_{N}$ is the gravitational constant in $(d+1)$ dimension and $\gamma_{\mathcal{A}}$ is the $(d-1)$ dimensional minimal-area hypersuface which extends into the bulk and has the same boundary $\partial \mathcal{A}$ of the subsystem $\mathcal{A}$.

Several computations of the HEE has been performed using the Ryu-Takayanagi prescription, and they are in good agreement with CFT results. For example, using standard techniques, one can compute the EE for a subsystem of length $l$ in a 2D CFT, which is given by $S_{\mathcal{A}}=\frac{c}{3} \ln \frac{l}{\epsilon}$ where $c$ is the central charge of the CFT and $\epsilon$ is an UV cut-off [23]. Instead, using $\mathrm{AdS}_{3} / \mathrm{CFT}_{2}$, if we apply the Ryu-Takayanagi formula for the HEE, we get the same result, with $c=3 R / 2 G_{N}^{(3)}$, where $R$ is radius of curvature of $\operatorname{AdS}_{3}$ and $G_{N}^{(3)}$ is the three dimensional gravitational constant.

Now we calculate the entanglement entropy of the VGHS and study the effect of the higher derivative coupling term $\eta$ and the model parameter $\Sigma$ on its HEE. First, as a warm up exercise, we calculate the HEE for the VGHS in an AdS-Schwarzschild background. Since the necessary formulas were worked out in [7], we do not show them here, but for completeness reproduce them in appendix B. The strategy of the computation is standard. Having solved the coupled equations in the bulk and thus having found the gravity solution both in the superconducting phase as well as in the normal phase, we use the Ryu-Takayanagi prescription to determine the HEE for both the normal and the superconducting phases. For this we consider our subsystem $\mathcal{A}$ to be a straight strip residing on the boundary. The domain $-\frac{l}{2} \leq x \leq \frac{l}{2}$ and $0 \leq y \leq L_{0}$, defines the strip geometry on the boundary, where $l$ is the size of region $\mathcal{A}$ and $L_{0}$ is a regulator which we can later set to infinity. Now we parameterize the minimal surface $\gamma_{\mathcal{A}}$, which extends in the bulk, by $x=x(z)$ and calculate the area of this hypersurface using the metric of eq. (B.4). This is given as

$$
\operatorname{Area}\left(\gamma_{\mathcal{A}}\right)=L_{0} \int_{-l / 2}^{l / 2} \frac{d x}{z^{2}} \sqrt{1+\frac{z^{\prime}(x)^{2}}{f(z)}} .
$$


Minimization of this area functional yields,

$$
\frac{1}{z^{2}} \frac{1}{\sqrt{1+\frac{z^{\prime}(x)^{2}}{f(z)}}}=\frac{1}{z_{*}^{2}}
$$

where $z_{*}$ is the turning point of the minimal area such that $\left.z^{\prime}(x)\right|_{z=z_{*}}=0$. Finally, one can obtain the entanglement entropy [5] as

$$
S=\frac{\operatorname{Area}\left(\gamma_{\mathcal{A}}\right)}{4 G_{4}}=\frac{2 L_{0}}{4 G_{4}} \int_{\epsilon}^{z_{*}} d z \frac{z_{*}^{2}}{z^{2}} \frac{1}{\sqrt{\left(z_{*}^{4}-z^{4}\right) f(z)}}=\frac{2 L_{0}}{4 G_{4}}\left(s+\frac{1}{\epsilon}\right),
$$

with

$$
\frac{l}{2}=\int_{\epsilon}^{z_{*}} d z \frac{z^{2}}{\sqrt{\left(z_{*}^{4}-z^{4}\right) f(z)}}
$$

where in eq. (4.5) the first term $s$ is the finite part of entanglement entropy. We also see that the second term in this equation diverges as $\epsilon \rightarrow 0$ and $z=\epsilon$ defines the UV cutoff. Since the finite part $s$ does not depend on any cutoff, it is the quantity which is physically important. So in the rest of our calculations, we will only deal with the finite part of the entanglement entropy.

For comparison, We will first show the results for the condensate for AdS-Schwarzschild black hole background, using the formulas presented in appendix B. Figure 16 shows how the condensate grows as one decreases the temperature below the critical value of $T / \mu$ for $\Sigma=5$, where the red, green, blue, brown, orange, pink and cyan curves correspond to $\eta=$ $0.01,-0.01,-0.1,-0.5,-1,-3$, and -5 respectively. Figure 17 shows the behavior of condensate as a function of temperature for $\eta=-0.1$ where the red, green, blue, brown and orange curves correspond to $\Sigma=0,1,3,5$ and 7 respectively. For detail on the analysis of this model, see [7].

Keeping in mind that the dimensionless quantities here are $\frac{T}{\mu}, \frac{s}{\mu}$ and $l \mu$, we first examine how the HEE changes when we vary the temperature, while keeping the strip width fixed. We set $\frac{l}{2} \mu=1, \Sigma=5,2 \kappa^{2}=0.5$ and consider different values of $\eta$. The results are shown in figure 18 where the same color coding as figure 16 has been used, and the solid black curve denotes the HEE for the normal phase. For $\eta=0.01$, there is a discontinuity in the slope of $s$ at the critical value of $\frac{T}{\mu}$, which indicates a second order phase transition from normal to superconducting phase [6, 21].

As we decrease the value of $\eta$ from 0.01 we see that $s$ becomes multivalued near the critical value of $T / \mu$ and that there is a discontinuous jump in the value of $s$ at the transition point, which indicates a first order phase transition [6]. If we continue to decrease the value of $\eta$ the transition again becomes of second order. Indeed, from figure 18 we see that $\eta=-0.1,-0.5,-1$ give first order phase transitions, while $\eta=-5$ gives second order phase transition. Thus, like the free energy calculations, the HEE in the VGHS also tells us that for a fixed value of $\Sigma$ and $\kappa$ there exists a window in $\eta$ where the transition from the normal phase to the superconducting phase is of first order, but outside this window the transition is of second order. This agrees perfectly with our result on condensate as a function of temperature which is shown in figure 16. We notice that for a fixed value of 

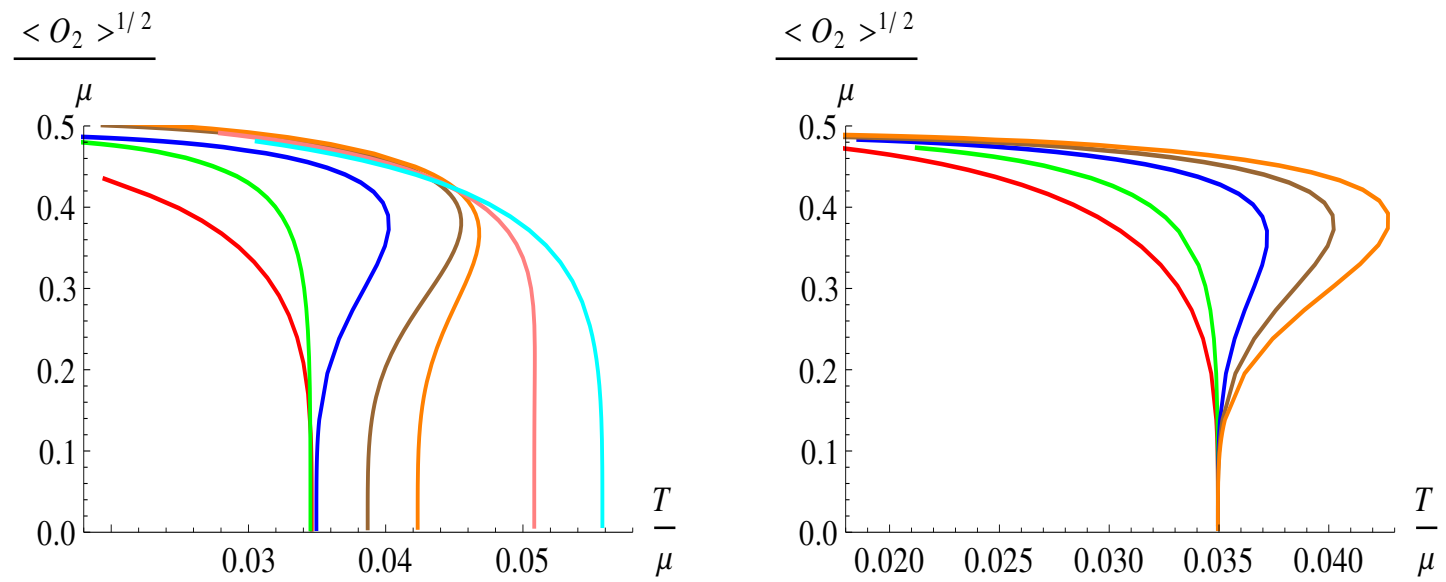

Figure 16. Variation of the condensate for dif- Figure 17. Variation of the condensate for ferent values of $\eta$ with fixed $\Sigma=5$ and $2 \kappa^{2}=$ different values of $\Sigma$ with fixed $\eta=-0.1$ and 0.5 for $4 \mathrm{D}$ AdS-Schwarzschild black hole back- $2 \kappa^{2}=0.5$ for $4 \mathrm{D}$ AdS-Schwarzschild black hole grounds. backgrounds.
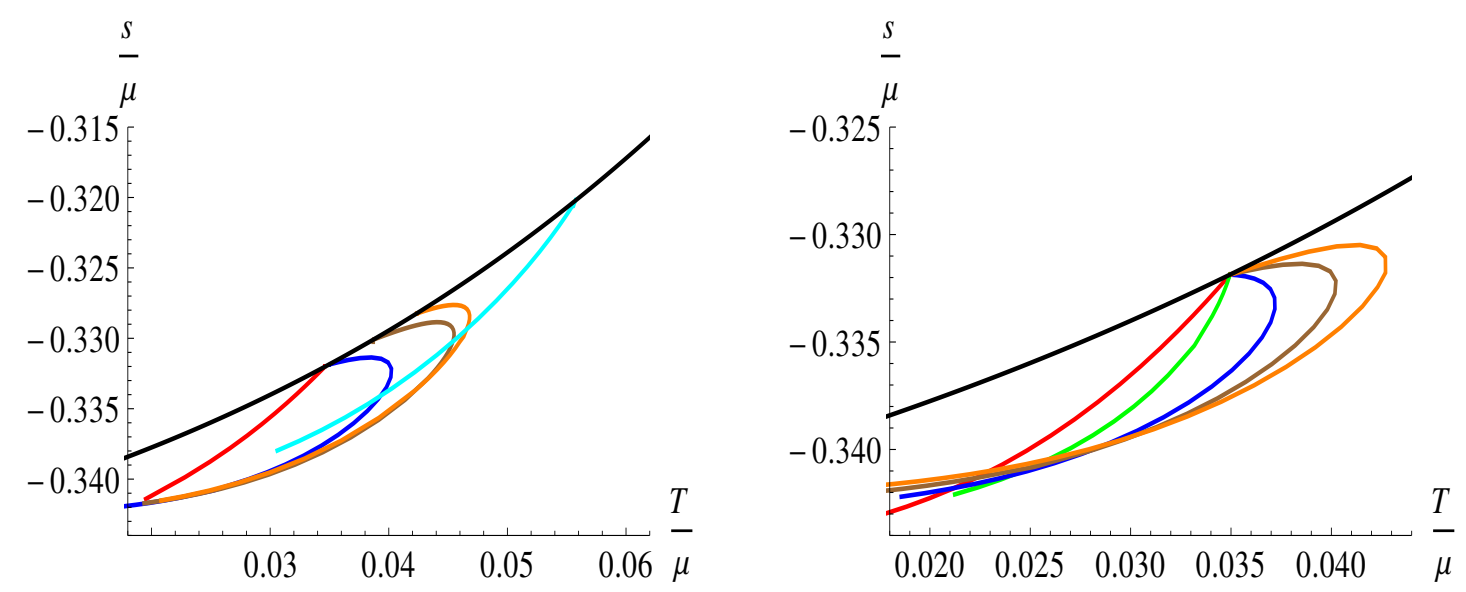

Figure 18. HEE for fixed $\frac{l}{2} \mu=1, \Sigma=5$ and Figure 19. HEE for fixed $\frac{l}{2} \mu=1, \eta=-0.1$, $2 \kappa^{2}=0.5$ for different values of $\eta$. $2 \kappa^{2}=0.5$ for different values of $\Sigma$.

the strip width, the superconducting solution always has lower entanglement entropy than the normal solution, consistent with our previous discussion. However for the VGHS in R-charged black hole backgrounds, which we momentarily turn to, we will find that this result can change, namely the HEE in the superconducting phase can be higher than that in the normal phase.

Now we will analyze the HEE as a function of $\Sigma$, for fixed $\eta$. This is shown in figure 19, where we have set $\frac{l}{2} \mu=1$ and $2 \kappa^{2}=0.5$. In figure 19 , the same color coding as in figure 17 has been used. We see that for $\Sigma=0$ and 1, the transition is second order, but if we increase the value of $\Sigma$, there is a discontinuous jump in $s$ after a certain value of $\Sigma$, indicating a first order transition. This implies that, for a fixed value of $\eta$ and $\kappa$, there exists a lower cut-off $\Sigma_{c}$ above which the phase transition is always of first order. This again agrees with our earlier findings. We have checked for a number of cases that as $\eta$ becomes more and more negative, the cut-off value $\Sigma_{c}$ increases. 


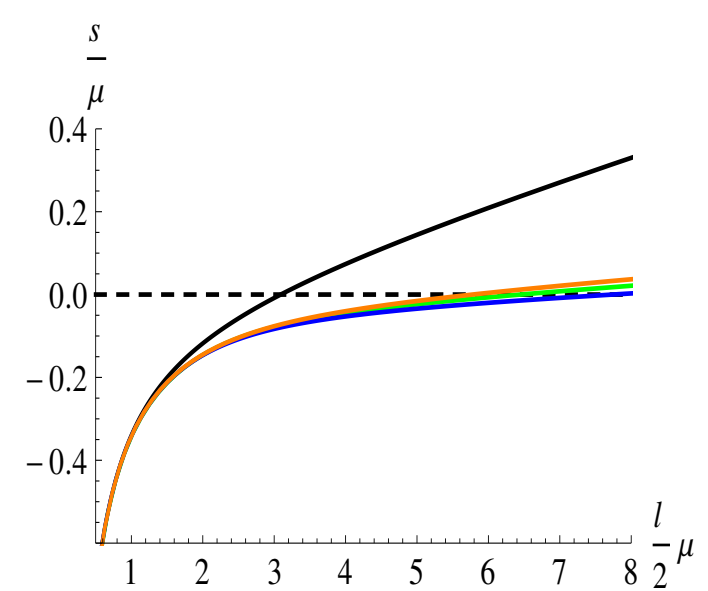

Figure 20. HEE for $T=0.5 T_{c}, \Sigma=5$ and Figure 21. HEE for $T=0.5 T_{c}, \eta=-0.1$ and $2 \kappa^{2}=0.5$ for different values of $\eta$.

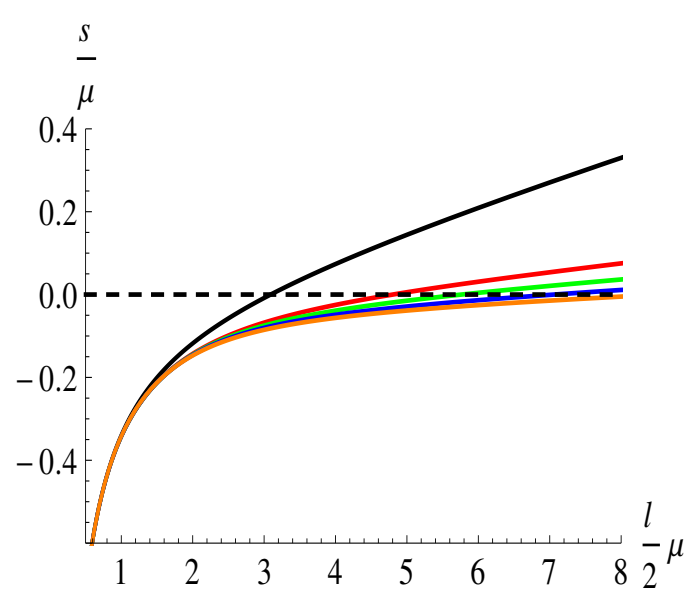

$2 \kappa^{2}=0.5$ for different values of $\Sigma$.

We record a further observation regarding the magnitudes of the entanglement entropy $s$. At a fixed temperature as we increase $\Sigma, s$ first decreases but if we continue to increase the value of $\Sigma$, at a certain point $s$ starts to increase. However, this behavior depends on temperature. For example, at $\frac{T}{\mu}=0.030$ the entanglement entropy for $\Sigma=0$ is greater than that for $\Sigma=3$. But at $\frac{T}{\mu}=0.020$ which is a relatively low temperature, the entanglement entropy for $\Sigma=0$ becomes less than that for $\Sigma=3$. However, we mention here that for very low temperatures, numerical calculations are not very trustworthy and therefore we refrain from making any exact statement here.

To complete the analysis, we have also calculated the behavior of the entanglement entropy $s$ as a function of strip width $l$, at a fixed temperature. This is shown in figures 20 and 21 , where we have set $T=0.5 T_{c}$ and $2 \kappa^{2}=0.5$. The solid black line denotes the HEE for normal phase. We see that for each case as we increase $l, s$ monotonically increases from a negative value and attains a positive value for large $l$.

We now turn to the computation of HEE in the VGHS in 4-D R-charged backgrounds, considered in section 2. We take the same metric ansatz with back reaction as in eq. (2.4) which we reproduce here for convenience

$$
d s^{2}=-g(r) H(r)^{-1 / 2} e^{-\chi(r)} d t^{2}+\frac{H(r)^{1 / 2}}{g(r)} d r^{2}+H(r)^{1 / 2} r^{2}\left(d x^{2}+d y^{2}\right)
$$

Now introducing $z=1 / r$, the above metric can be written as

$$
d s^{2}=-g(z) H(z)^{-1 / 2} e^{-\chi(z)} d t^{2}+\frac{H(z)^{1 / 2}}{z^{4} g(z)} d z^{2}+\frac{H(z)^{1 / 2}}{z^{2}}\left(d x^{2}+d y^{2}\right)
$$

Here, $z=1$ corresponds to the the horizon and $z=0$ to the boundary. We can calculate the HEE for the superconducting and the normal phase in the same way as we did with the AdS-Schwarzschild background. For this we again take our subsystem $\mathcal{A}$, residing on the boundary, to be a straight strip and define its domain by $-\frac{l}{2} \leq x \leq \frac{l}{2}$ and $0 \leq y \leq L_{0}$. Parameterizing the minimal surface $\gamma_{\mathcal{A}}$ by $x=x(z)$, we first calculate the area of this 
hypersurface,

$$
\operatorname{Area}\left(\gamma_{\mathcal{A}}\right)=L_{0} \int_{-l / 2}^{l / 2} \frac{d x}{z^{2}} \sqrt{H(z)\left(1+\frac{z^{\prime}(x)^{2}}{z^{2} g(z)}\right)} .
$$

When we minimize the above area functional, we get the equation for the minimal surface

$$
\frac{\sqrt{H(z)}}{z^{2} \sqrt{1+\frac{z^{\prime}(x)^{2}}{z^{2} g(z)}}}=\frac{\sqrt{H\left(z_{*}\right)}}{z_{*}^{2}}
$$

where, as before, $z_{*}$ represents the turning point of the minimal surface such that $\left.z^{\prime}(x)\right|_{z=z_{*}}=0$. Finally, one can write down the entanglement entropy [5] as

$$
S=\frac{\operatorname{Area}\left(\gamma_{\mathcal{A}}\right)}{4 G_{4}}=\frac{2 L_{0}}{4 G_{4}} \int_{\epsilon}^{z_{*}} d z \frac{z_{*}^{2}}{z^{3}} \frac{H(z)}{\sqrt{\left(z_{*}^{4} H(z)-z^{4} H\left(z_{*}\right)\right) g(z)}}=\frac{2 L_{0}}{4 G_{4}}\left(s+\frac{1}{\epsilon}\right),
$$

with

$$
\frac{l}{2}=\int_{\epsilon}^{z_{*}} d z \frac{z \sqrt{H\left(z_{*}\right)}}{\sqrt{\left(z_{*}^{4} H(z)-z^{4} H\left(z_{*}\right)\right) g(z)}}
$$

In eq. (4.11) the first term $s$ represents the finite part of the EE. Like our previous case with the AdS Schwarzschild background, we will only concentrate on the computation of the physically relevant finite part $s$ of the EE.

First we study the behavior of the HEE with temperature, keeping the strip width fixed. We set $\frac{l}{2} \mu=1, \eta=-0.1,2 \kappa^{2}=0.3$ and take different values of $\Sigma$. The results are shown in figure 22 where the black curve denotes the HEE for the normal phase. The curves with red, green, blue, brown and orange color correspond to $\Sigma=1,5,7,10$ and 15 respectively. The most important observation here is that the HEE in the superconducting phase is greater than that for the normal phase, which seems to contradict expected behavior. However, we remind the reader that as pointed out in the introduction, this may not have anything to do with the number of degrees of freedom of the system. The issue needs further understanding, and a computation in lines of [22] might be more helpful here, but we do not undertake such an analysis in this paper. However, we point out that as expected, a calculation of the free energy here shows that in the superconducting phase it is smaller than that in the normal phase. This situation is not repeated in the VGHS in five dimensional R-charged backgrounds, where we find the HEE in the superconducting phase is smaller than the normal phase. Therefore, the higher magnitude of the HEE in our set up seems to be a special property of four dimensional R-charged black hole background.

At this point we are unable to explain this behavior of HEE for the VGHS in four dimensional R-charge backgrounds (as we elaborate shortly). However, the order of the phase transition is clear from the figure and it is consistent with our previous result on condensate as a function of temperature. For $\Sigma=1,5$ and 7 , the slope in the HEE shows a discontinuity at the critical value of $\frac{T}{\mu}$, indicating a second order phase transition. But as one increases the value of $\Sigma$ from $\Sigma=7$, the HEE becomes multivalued near the critical value of $T / \mu$, showing a discontinuous jump in $s$, which indicates a first order phase transition. 


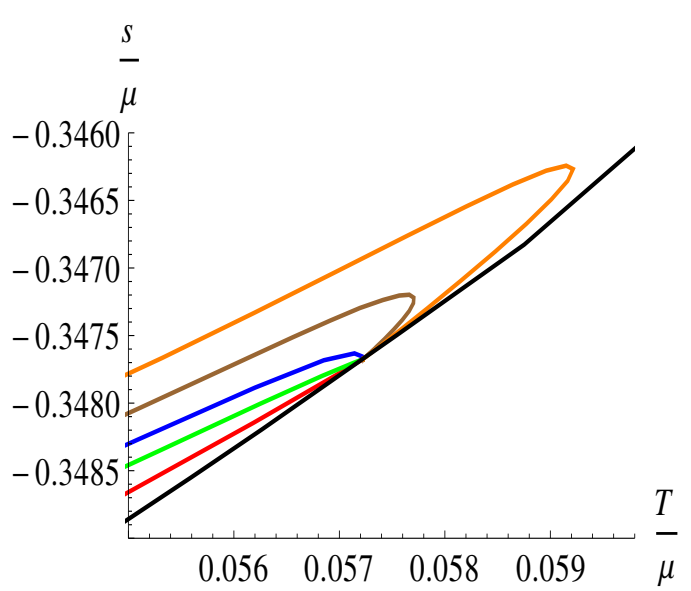

Figure 22. HEE for fixed $\frac{l}{2} \mu=1, \eta=-0.1$ and Figure 23. HEE for fixed $\frac{l}{2} \mu=1, \Sigma=15$ and $2 \kappa^{2}=0.3$ for different $\Sigma$.
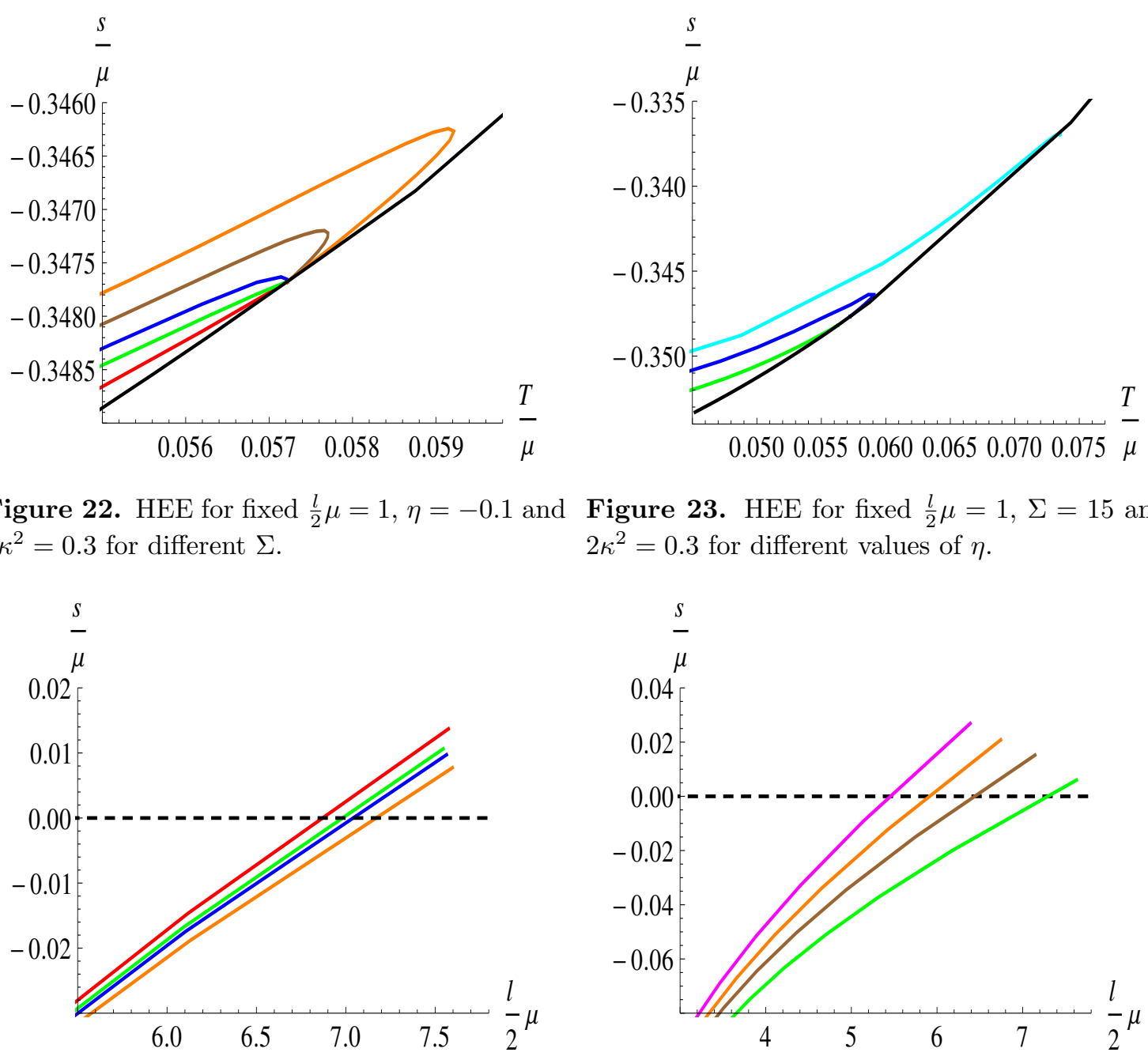

$2 \kappa^{2}=0.3$ for different values of $\eta$.

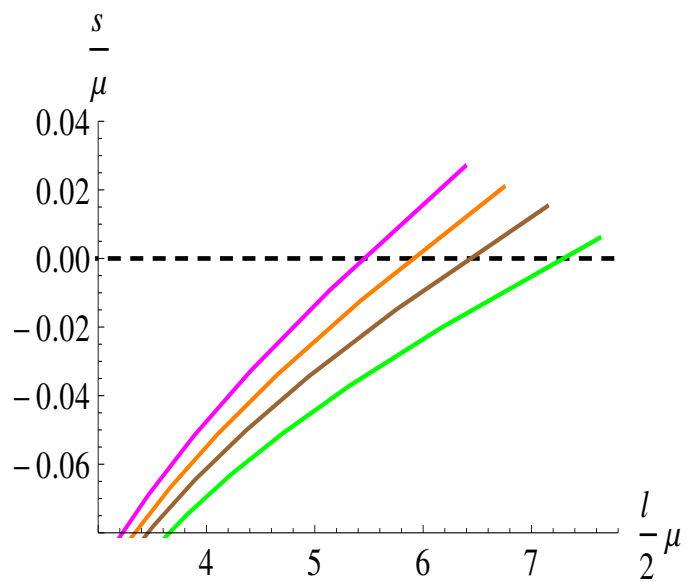

Figure 24. HEE for $T=0.5 T_{c}, \eta=-0.1$ Figure 25. HEE for $T=0.5 T_{c}, \Sigma=15$ and $2 \kappa^{2}=0.3$ for different values of $\Sigma$. Red, and $2 \kappa^{2}=0.3$ for different values of $\eta$. Green, green, blue and orange colors correspond to $\Sigma=$ brown, orange and magenta correspond to $\eta=$ $1,5,7$ and 15 respectively.

Now taking $\eta$ as the varying parameter, and fixing $\Sigma$, we show the HEE in figure 23, where the black curve represent the HEE in the normal phase. Here we set $\frac{l}{2} \mu=1$, $\Sigma=15,2 \kappa^{2}=0.3$. The curves with green, blue and cyan colors correspond to $\eta=$ $-0.01,-0.1$ and -3 respectively. The window of first order transitions should be obvious, but again, the HEE in the superconducting phase seems to be greater than that in the normal phase.

It is difficult to pinpoint the physical reason for this behavior as the computations are entirely numerical. If we set $H=1$ in the 4-D R-charged background, we recover the usual behavior for the HEE, as in AdS-Schwarzschild examples. Although this would suggest that the difference in the R-charged background is due to the $H(r)$ term in the metric, one has to be careful before drawing any conclusion. This is because we have checked that the nature of the functions $g(r), H(r)$ and $\chi(r)$ are all qualitatively similar in the VGHS in 
four as well as five dimensional R-charged backgrounds. The fact that the HEE behaves differently only in four dimensions is possibly due to the different nature of the coupled differential equations in these systems. We do not have a better understanding of this as of now.

We have also studied the behavior of entanglement entropy as a function of the strip width $l$ at a fixed temperature. The results are shown in figure 24 and 25 where we have set $T=0.5 T_{c}$ and $2 \kappa^{2}=0.3$. The behavior of the HEE with $l$ is qualitatively similar to that with the AdS-Schwarzschild background we have just studied. We find that for each case as we increase $l, s$ monotonically increases from negative values and attains a positive values for large $l$.

\section{Entangling temperature of holographic superconductors}

While discussing HEE, it is very interesting to ask whether there exists here a "first law of thermodynamics." Recently, this question has been discussed in [25], where it is shown that for a small subsystem, the change of the entanglement entropy is proportional to the change of the energy of the subsystem and the proportionality constant, which is given by the size of the entangling region, is interpreted as the inverse of the entangling temperature. The procedure to establish this is to calculate the entanglement entropy and energy for the excited state of a d-dimensional boundary CFT. The dual gravitational picture of this excited state is the deformed AdS space. Since we want to calculate the entangling temperature in our model of holographic superconductors, we will consider AdS black holes with scalar hair as the deformed AdS space and as mentioned above this would correspond to excited state of the boundary CFT. Then by calculating the change in entanglement entropy $(\Delta S)$ and the change in energy $(\Delta E)$ of the boundary CFT due to this deformation, one can calculate the entangling temperature.

The computation of entangling temperature in the context of holography involves a number of steps. We will not mention the details here but refer the interested reader to [27]. As considered in [25, 27], we choose our ground state in the CFT to be dual to four dimensional pure AdS with metric

$$
d s^{2}=\frac{1}{z^{2}}\left(-d t^{2}+d z^{2}+d x^{2}+d y^{2}\right)
$$

and the entanglement entropy of the ground state with a subsystem of straight strip of width $(l)$ is given by

$$
S_{E}^{(0)}=\frac{2 L_{0}}{4 G_{4}}\left[\frac{1}{\epsilon}-\frac{2 \pi}{l}\left(\frac{\Gamma\left(\frac{3}{4}\right)}{\Gamma\left(\frac{1}{4}\right)}\right)^{2}\right]
$$

The excited state of the boundary CFT (the superconducting phase) in our case will be described by the following metric in the bulk,

$$
d s^{2}=\frac{1}{z^{2}}\left(-f(z) e^{-\chi(z)} d t^{2}+\frac{d z^{2}}{f(z)}+d x^{2}+d y^{2}\right)
$$




\begin{tabular}{|c|c|c|c|c|c|c|c|}
\hline$\Sigma \backslash a_{i}$ & $a_{3}$ & $a_{4}$ & $a_{5}$ & $a_{6}$ & $a_{7}$ & $a_{8}$ & $a_{9}$ \\
\hline 0 & -20.261 & 89.562 & -166.732 & 148.364 & -51.171 & -7.991 & 7.229 \\
\hline 1 & -38.439 & 220.521 & -524.026 & 639.927 & -412.217 & 123.640 & -10.404 \\
\hline 3 & -76.493 & 584.037 & -1851.370 & 3124.140 & -2967.180 & 1501.940 & -316.077 \\
\hline 5 & -93.975 & 781.032 & -2660.490 & 4786.60 & -4814.06 & 2565.35 & -565.479 \\
\hline 7 & -106.590 & 943.375 & -3374.180 & 6321.33 & -6578.09 & 3608.8 & -815.656 \\
\hline
\end{tabular}

Table 1. Coefficients of $f(z)$, for fixed $\eta=-0.1$

The above metric can be considered as a thermal deformation of the pure AdS geometry (5.1) such that the boundary theory which now has a non-zero temperature corresponds to an excited state. Our strategy here is to compute the form of $f(z)$ and $\chi(z)$ numerically at a particular temperature (below $T_{c}$, so that we are in the superconductor phase) and calculate the change in entanglement entropy caused by this deformation. In order to calculate the change in energy of the subsystem, it is useful to cast the metric (5.3) in Fefferman-Graham coordinates,

$$
d s^{2}=\frac{1}{z^{2}}\left(d z^{2}+g_{\mu \nu} d x^{\mu} d x^{\nu}\right)
$$

where $g_{\mu \nu}=\eta_{\mu \nu}+h_{\mu \nu}(x, z)$ with

$$
h_{\mu \nu}(x, z)=h_{\mu \nu}^{(0)}(x)+z^{2} h_{\mu \nu}^{(2)}(x)+z^{3} h_{\mu \nu}^{(3)}(x)+\cdots
$$

From the expansion it is clear that $h_{\mu \nu}(x, z)$ contains the information about the excited state.

Now at a particular temperature, we fit the numerical solution of $f(z)$ and $\chi(z)$ with the polynomials

$$
\begin{aligned}
& f(z)=1+a_{3} z^{3}+a_{4} z^{4}+\cdots \\
& \chi(z)=A_{2} z^{2}+A_{3} z^{3}+A_{4} z^{4}+\cdots
\end{aligned}
$$

and calculate the coefficients $a_{k}$ and $A_{k}$ for all $k$. Note that the form of $f(z)$ and $\chi(z)$ depend on the higher derivative coupling constant $\eta$, and that the model parameter $\Sigma$, so the coefficients $a_{k}$ and $A_{k}$ will also change accordingly. For example, for the AdSSchwarzchild black hole background, the polynomial coefficients that fit the curve $f(z)$ and $\chi(z)$ for $T=0.5 T_{c}$ and $\eta=-0.1$, with the backreaction parameter $2 \kappa^{2}=0.5$ are given in tables (1) and (2). ${ }^{8}$

Similarly, the coefficients $a_{i}$ and $A_{i}$ from polynomial fitting of $f(z)$ and $\chi(z)$ at $T=0.5 T_{c}, 2 \kappa^{2}=0.5$, and $\Sigma=5$, for different values of $\eta$, are shown in the following tables (3) and (4):

\footnotetext{
${ }^{8}$ For ease of presentation, we have truncated some of the numbers that appear in the following tables. An exact fit obtained by using a standard MATHEMATICA routine provides slightly more precise values.
} 


\begin{tabular}{|c|c|c|c|c|c|c|}
\hline$\Sigma \backslash A_{i}$ & $A_{2}$ & $A_{3}$ & $A_{4}$ & $A_{5}$ & $A_{6}$ & $A_{7}$ \\
\hline 0 & -0.830 & 9.744 & -0.151 & -31.346 & 36.772 & -12.844 \\
\hline 1 & -2.790 & 34.691 & -68.335 & 46.345 & -2.647 & -5.802 \\
\hline 3 & -6.319 & 87.75 & -262.584 & 353.392 & -228.937 & 58.165 \\
\hline 5 & -7.019 & 106.638 & -345.949 & 501.759 & -348.364 & 94.374 \\
\hline 7 & -6.569 & 117.047 & -405.317 & 617.857 & -446.948 & 125.367 \\
\hline
\end{tabular}

Table 2. Coefficients of $\chi(z)$, for fixed $\eta=-0.1$

\begin{tabular}{|c|c|c|c|c|c|c|c|}
\hline$\eta \backslash a_{i}$ & $a_{3}$ & $a_{4}$ & $a_{5}$ & $a_{6}$ & $a_{7}$ & $a_{8}$ & $a_{9}$ \\
\hline-0.01 & -48.4672 & 303.385 & -781.153 & 1051.61 & -778.868 & 296.878 & -44.380 \\
\hline-0.1 & -93.9752 & 781.032 & -2660.49 & 4786.6 & -4814.06 & 2565.35 & -565.479 \\
\hline-0.5 & -72.6527 & 585.099 & -1970.95 & 3531.54 & -3548.66 & 1892.4 & -417.783 \\
\hline-1 & -51.9212 & 373.551 & -1157.72 & 1936.37 & -1834.96 & 930.064 & -196.39 \\
\hline
\end{tabular}

Table 3. Coefficients of $f(z)$, for fixed $\Sigma=5$

\begin{tabular}{|c|c|c|c|c|c|c|}
\hline$\eta \backslash A_{i}$ & $A_{2}$ & $A_{3}$ & $A_{4}$ & $A_{5}$ & $A_{6}$ & $A_{7}$ \\
\hline-0.01 & -3.997 & 49.748 & -108.541 & 92.630 & -27.095 & -1.053 \\
\hline-0.1 & -7.019 & 106.638 & -345.949 & 501.759 & -348.364 & 94.374 \\
\hline-0.5 & -4.188 & 69.8242 & -229.024 & 334.125 & -233.174 & 63.484 \\
\hline-1 & -2.842 & 43.6855 & -131.069 & 176.526 & -114.738 & 29.323 \\
\hline
\end{tabular}

Table 4. Coefficients of $\chi(z)$, for fixed $\Sigma=5$

Now by substituting the form of $f(z)$ and $\chi(z)$ into (5.3), we can cast it into the form of (5.4) and therefore can calculate the coefficients $h_{\mu \nu}^{(0)}(x), h_{\mu \nu}^{(2)}(x), h_{\mu \nu}^{(3)}(x)$ etc. Assuming that $h_{\mu \nu}^{(n)} l^{n} \ll 1$ throughout our calculation and following [27], we find the increase in entanglement entropy of the excited state with respect to the ground state as

$$
\Delta S_{E}=\frac{1}{4 G_{4}} \int_{0}^{z_{*}} d z\left(\Gamma^{(0)}+\Gamma^{(2)} z^{2}+\Gamma^{(3)} z^{3}+\cdots\right)
$$

where

$$
\begin{aligned}
\int_{\epsilon}^{z_{*}} \Gamma^{(n)} r^{n}= & \frac{1}{(1-n) \epsilon^{1-n}} \int d x\left(\operatorname{Tr}\left(h_{a b}^{(n)}\right)-h_{11}^{(n)}\right) \\
& -F(2,2-n) \frac{l^{n-1}}{2^{n-1} a_{\zeta}^{n-1}} \int d x\left(\operatorname{Tr}\left(h_{a b}^{(n)}\right)-\frac{2}{n+1} h_{11}^{(n)}\right)
\end{aligned}
$$

with

$$
F(m, n)=\frac{{ }_{2} F_{1}\left(\frac{1}{2}, \frac{1-n}{2 m}, \frac{2 m+1-n}{2 m}, 1\right)}{n-1}, a_{\zeta}=\frac{\sqrt{\pi} \Gamma\left(\frac{3}{4}\right)}{\Gamma\left(\frac{1}{4}\right)}
$$

Here ${ }_{2} F_{1}$ is the Hypergeometric function and $z_{*}$ is the turning point with pure AdS geometry. Now, using the prescription of $[29,30]$, the energy momentum tensor of the excited 

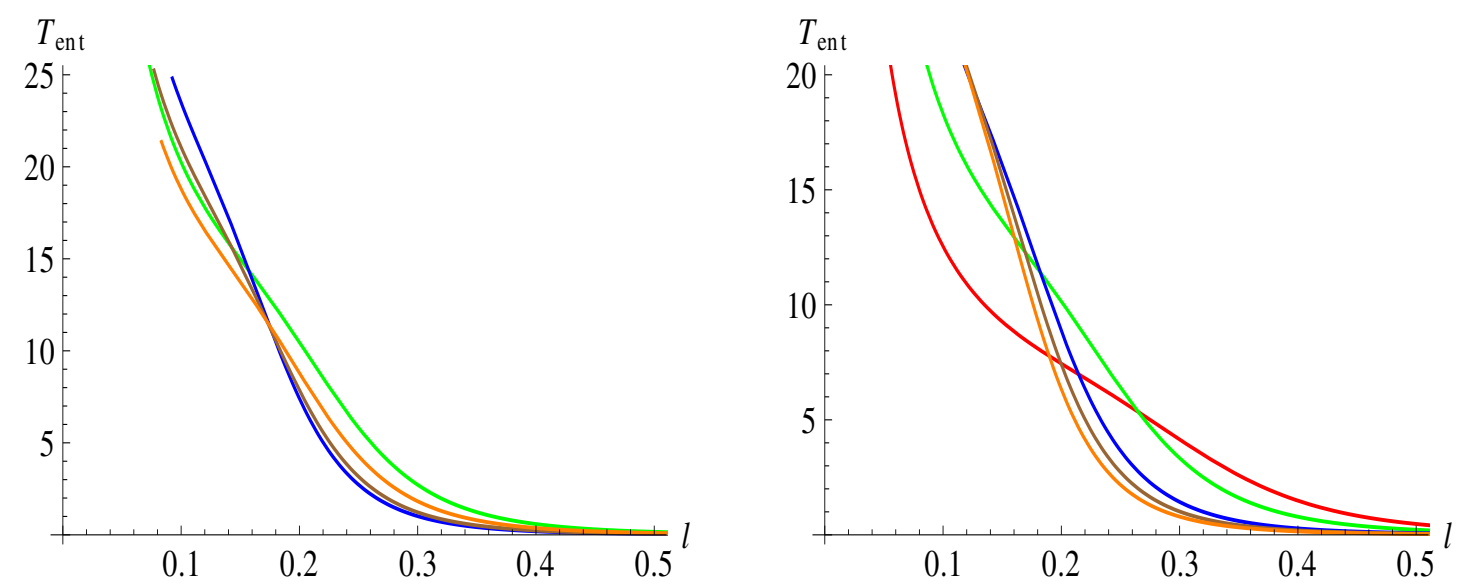

Figure 26. $T_{\text {ent }}$ vs $l$ at $T=0.5 T_{c}, \Sigma=5$ and Figure 27. $T_{\text {ent }}$ vs $l$ at $T=0.5 T_{c}, \eta=-0.1$ $2 \kappa^{2}=0.5$ for different values of $\eta$. and $2 \kappa^{2}=0.5$ for different $\Sigma$.

state in the dual CFT side is given as

$$
\left\langle T_{\mu \nu}\right\rangle=\frac{3}{16 \pi G_{4}} h_{\mu \nu}^{(3)}
$$

We use the above expression to calculate the increase in energy of the excited state as

$$
\Delta E=\int d^{2} x\left\langle\Delta T_{t t}\right\rangle
$$

From the above discussion, it is clear that $\Delta E$ is always proportional to $l$. Using (5.7) and (5.10), we calculate the entangling temperature as $T_{\text {ent }}=\frac{\Delta E}{\Delta S_{E}}$. In figure 26 we have shown the variation of entangling temperature $T_{\text {ent }}$ as a function of the strip width $l$ for different values of $\eta$. The green, blue, brown and orange curves correspond to $\eta=$ $-0.01,-0.1,-0.5$ and -1 , respectively. Here we have fixed $T=0.5 T_{c}, \Sigma=5$ and $2 \kappa^{2}=0.5$. We note that as one decreases the strip width, $T_{\text {ent }}$ increases and diverges as $l \rightarrow 0$. Physically, this corresponds to the fact that at zero strip width, there is no entanglement.

Qualitatively similar behavior for $T_{\text {ent }}$ was observed in [25], where the authors found $T_{\text {ent }} \propto 1 / l$ for AdS-Schwarzschild black hole (without any scalar hair). In our case, there are a few differences. First, for the case of the non-hairy AdS-Schwarzschild black hole, only $h_{\mu \nu}^{(3)}(x)$ is non zero and therefore $T_{\text {ent }}$ is always proportional to $1 / l$. For the VGHS, higher order terms in eq. (5.5) can be nonzero and therefore can modify the $T_{\text {ent }} \propto 1 / l$ relation. Indeed this is what we see in figure 26 . We can also calculate the departure of $T_{\text {ent }}$ from the $1 / l$ behavior that appear in the four dimensional non-hairy AdS-Schwarzschild black hole case. This is calculated as $\frac{T_{\text {ent }}^{(1)}-T_{\text {ent }}^{(2)}}{T_{\text {ent }}^{(1)}}$, where $T_{\text {ent }}^{(1)}$ and $T_{\text {ent }}^{(2)}$ are the entangling temperatures for the non-hairy AdS-Schwarzschild case and the VGHS in the AdS-Schwarzschild cases, respectively. From eq. (5.7), one can see that there is a contribution to the entanglement entropy not only from $\Gamma^{(0)}, \Gamma^{(2)}$ and $\Gamma^{(3)}$, but also from the higher order terms. This is because while expanding $h_{\mu \nu}(x)$ in eqn (33), one needs to consider the terms beyond 
$h_{\mu \nu}^{(3)}(x)$. The appearance of these extra terms is what modifies the behavior of $T_{\text {ent }}$. For $\eta=-0.01, \Sigma=5$ and $T=0.5 T_{c}$ we find a departure of around $17 \%$ for $l=0.05$ and $37 \%$ for $l=0.1$. However, the departure from $T_{\text {ent }} \propto 1 / l$ is not very large near criticality. For small value of $l$, say $l \backsim 0.25$, we find a departure of around $3 \%$ and for $l \backsim 0.6$ we find a departure of around $15 \%$ near $T_{c}$ for the same values of $\eta$ and $\Sigma$.

We also extend the analysis for different values of $\Sigma$ for fixed $\eta$ and $\kappa$, at $T=0.5 T_{c}$. This is shown in figure 27 where the red, green, blue, brown and orange curves correspond to $\Sigma=0,1,3,5$ and 7 , respectively.

For the VGHS in R-charge backgrounds, we ran into some difficulties with the above procedure. This is due to the fact that fitting polynomials for $f(z)$ and $\chi(z)$ are difficult to obtain very precisely. Due to this, the entangling temperature could not be calculated properly. We will not discuss this issue further.

\section{Conclusions and discussions}

In this concluding section, we will summarize our main results. First, we have constructed a very general class of phenomenological models for holographic superconductors in single R-charged black hole backgrounds, in four and five dimensions, including back reaction effects. The VGHS models constructed in this paper correspond to supergravity backgrounds of rotating brane configurations, and hence non-trivially extend the ones considered in [7] for the AdS-Schwarzschild case. We find that our models predict a rich phase structure in the parameter space, with a window of first order phase transitions. ${ }^{9}$ As pointed out in the text, this might be phenomenologically important in the understanding of the strongly coupled behavior of superconductors. In the probe limit, the phase diagram of our model is qualitatively shown in figure 1. Admittedly, the results contained in this paper cannot be used to understand realistic physical phenomena as of now, but these further our understanding of phase transitions in holographic scenarios, and we only hope that they should be useful in future experiments.

Next, we studied holographic entanglement entropy for our model, and found that the HEE precisely captures the information about the phase transitions alluded to above. In the window of parameters where a first order phase transition is predicted by a calculation of the free energy, the HEE for the superconducting phase is multi valued, and is single valued outside. However we find that in the four dimensional example that we have worked out, the HEE seems to be higher in the superconducting phase, contrary to results that appear in the literature. Since the results are completely numerical, it is difficult to pinpoint the exact reason for this.

Finally, we studied the entangling temperature for generalized holographic superconductors in the AdS Schwarzschild background. We found that the temperature shows deviation from a pure AdS background, and that these are dependent on the model parameters. We were unable to perform this calculation in R-charged backgrounds, as it was

\footnotetext{
${ }^{9}$ For AdS-soliton backgrounds appropriate for studying insulator-superconductor phase transitions, we find that the VGHS does not show any such window.
} 
difficult to obtain exact fits to the metric components here. This case needs to be further investigated.

It will be interesting to calculate the optical response properties of the VGHS in Rcharge backgrounds, analogous to what was done in $[7,15]$. It might also be useful to consider different types of higher derivative couplings in holographic models. We leave these issues for a future publication.

\section{Acknowledgments}

The work of SM is supported by grant no. 09/092(0792)-2011-EMR-1 from CSIR, India.

\section{A Details of 5-D single R-charged black hole backgrounds}

In this appendix, we present the details of our calculations for holographic superconductors in 5-D R-charged black hole backgrounds. We start with the action

$$
\begin{aligned}
S= & \int \mathrm{d}^{5} x \sqrt{-g}\left[\frac{1}{2 \kappa^{2}}\left(R+4\left(H^{2 / 3}+2 H^{-1 / 3}\right)\right)-\frac{H^{4 / 3}}{8} F_{\mu \nu} F^{\mu \nu}-\frac{1}{3} \frac{(\partial H)^{2}}{H^{2}}\right. \\
& \left.-\frac{1}{2}|D \tilde{\Psi}|^{2}-\frac{1}{2} m^{2}|\tilde{\Psi}|^{2}-\frac{\eta}{2}\left|F_{\mu \nu} D^{\nu} \tilde{\Psi}\right|^{2}\right]
\end{aligned}
$$

Now writing the charged scalar field as $\tilde{\Psi}=\Psi e^{i \alpha}$ and following section 2, the action of eq. (A.1) can be generalized as

$$
\begin{aligned}
S= & \int \mathrm{d}^{5} x \sqrt{-g}\left[\frac{1}{2 \kappa^{2}}\left(R+4\left(H^{2 / 3}+2 H^{-1 / 3}\right)\right)-\frac{H^{4 / 3}}{8} F_{\mu \nu} F^{\mu \nu}-\frac{1}{3} \frac{(\partial H)^{2}}{H^{2}}\right. \\
& -\frac{\left(\partial_{\mu} \Psi\right)^{2}}{2}-\frac{\eta}{2} F_{\mu \nu} \partial^{\nu} \Psi F^{\mu \sigma} \partial_{\sigma} \Psi-\frac{m^{2} \Psi^{2}}{2}-\frac{|\mathrm{G}(\Psi)|(\partial \alpha-q A)^{2}}{2} \\
& \left.-\frac{\eta}{2}|\mathrm{~K}(\Psi)|\left(F^{\mu \nu}\left(\partial_{\nu} \alpha-q A_{\nu}\right)\right)^{2}\right]
\end{aligned}
$$

For 5-D background we will consider the following ansatz

$$
d s^{2}=-g(r) H(r)^{-2 / 3} e^{-\chi(r)} d t^{2}+H(r)^{1 / 3} r^{2}\left(d x^{2}+d y^{2}+d z^{2}\right)+H(r)^{1 / 3} \frac{d r^{2}}{g(r)}
$$

Equation of motion for the scalar field $\Psi$

$$
\begin{array}{r}
\Psi^{\prime \prime}\left(1-\eta e^{\chi} H^{1 / 3} \Phi^{\prime 2}\right)+\frac{H e^{\chi} \Phi^{2}}{2 g^{2}} \frac{d \mathrm{G}(\Psi)}{d \Psi}-\frac{\eta H^{4 / 3} e^{2 \chi} \Phi^{2} \Phi^{\prime 2}}{2 g^{2}} \frac{d \mathrm{~K}(\Psi)}{d \Psi} \\
-\eta H^{1 / 3} e^{\chi} \Psi^{\prime}\left(\frac{g^{\prime} \Phi^{\prime 2}}{g}+\frac{\chi^{\prime} \Phi^{\prime 2}}{2}+\frac{H^{\prime} \Phi^{\prime 2}}{3 H}+\frac{3 \Phi^{\prime 2}}{r}+2 \Phi^{\prime} \Phi^{\prime \prime}\right) \\
-\frac{m^{2} H^{1 / 3} \Psi}{g}+\Psi^{\prime}\left(\frac{3}{r}+\frac{g^{\prime}}{g}-\frac{\chi^{\prime}}{2}\right)=0
\end{array}
$$


Equation of motion for the zeroth component of the gauge field

$$
\begin{gathered}
\Phi^{\prime \prime}\left(1-\frac{2 \eta e^{\chi} \Phi^{2} \mathrm{~K}(\Psi)}{g H^{2 / 3}}+\frac{2 \eta g \Psi^{\prime 2}}{H^{5 / 3}}\right)-\Phi\left(\frac{2 \mathrm{G}(\Psi)}{g H}+\frac{2 \eta e^{\chi} \Phi^{\prime 2} \mathrm{~K}(\Psi)}{g H^{2 / 3}}\right) \\
+\frac{2 \eta g \Psi^{\prime 2} \Phi^{\prime}}{H^{5 / 3}}\left(\frac{g^{\prime}}{g}+\frac{\chi^{\prime}}{2}+\frac{H^{\prime}}{3 H}+\frac{3}{r}+\frac{2 \Psi^{\prime \prime}}{\Psi^{\prime}}\right)+\Phi^{\prime}\left(\frac{3}{r}+\frac{2 H^{\prime}}{H}+\frac{\chi^{\prime}}{2}\right) \\
+\frac{2 \eta e^{\chi} \mathrm{K}(\Psi) \Phi^{2} \Phi^{\prime}}{g H^{2 / 3}}\left(\frac{g^{\prime}}{g}-\frac{\mathrm{K}(\Psi)^{\prime}}{\mathrm{K}(\Psi)}-\frac{3 \chi^{\prime}}{2}-\frac{3}{r}-\frac{4 H^{\prime}}{3 H}\right)=0
\end{gathered}
$$

H-filed equation of motion

$$
H^{\prime \prime}+H^{\prime}\left(\frac{3}{r}+\frac{g^{\prime}}{g}-\frac{\chi^{\prime}}{2}-\frac{H^{\prime}}{H}\right)+\frac{e^{\chi} H^{3} \Phi^{\prime 2}}{2 g}+\frac{4 H}{2 \kappa^{2} g}(H-1)=0
$$

Similarly the Einstein equations give

$$
\begin{aligned}
& g^{\prime}+\frac{2 g}{r}-\frac{4 r}{3}(H+2)+\frac{r g^{\prime} H^{\prime}}{6 H}+\frac{g H^{\prime}}{H}-\frac{5 r g H^{\prime 2}}{18 H^{2}}+\frac{r g H^{\prime \prime}}{3 H} \\
& +2 \kappa^{2} r\left(\frac{H e^{\chi} \Phi^{2} \mathrm{G}(\Psi)}{6 g}+\frac{H^{1 / 3} m^{2} \Psi^{2}}{6}+\frac{g H^{\prime 2}}{9 H^{2}}+\frac{\eta g H^{1 / 3} e^{\chi} \Phi^{\prime 2} \Psi^{\prime 2}}{6}+\frac{e^{\chi} H^{2} \Phi^{\prime 2}}{12}\right. \\
& \left.-\frac{\eta H^{4 / 3} e^{2 \chi} \Phi^{2} \Phi^{\prime 2} \mathrm{~K}(\Psi)}{2 g}+\frac{g \Psi^{\prime 2}}{6}\right)=0 \\
& 2 \kappa^{2} r\left(\frac{H e^{\chi} \Phi^{2} \mathrm{G}(\Psi)}{3 g^{2}}+\frac{2 H^{\prime 2}}{9 H^{2}}-\frac{\eta H^{4 / 3} e^{2 \chi} \Phi^{2} \Phi^{\prime 2} \mathrm{~K}(\Psi)}{3 g^{2}}+\frac{\Psi^{\prime 2}}{3}-\frac{\eta H^{1 / 3} e^{\chi} \Phi^{\prime 2} \Psi^{\prime 2}}{3}\right) \\
& \chi^{\prime}\left(1+\frac{r H^{\prime}}{6 H}\right)+\frac{H^{\prime}}{H}-\frac{2 r H^{\prime} 2}{9 H^{2}}+\frac{r H^{\prime \prime}}{3 H}=0
\end{aligned}
$$

here again prime denotes a derivative with respect to $\mathrm{r}$ and also $\mathrm{r}$ dependence of each variable is suppressed. The hawking temperature for background with the metric (A.3) is given by

$$
T_{H}=\left.\frac{g^{\prime}(r) e^{-\chi(r) / 2}}{4 \pi \sqrt{H(r)}}\right|_{r=r_{h}}
$$

To solve these five coupled differential equations we impose the following boundary conditions

$$
\Phi\left(r_{h}\right)=0, \quad \Psi^{\prime}\left(r_{h}\right)=\frac{m^{2} H\left(r_{h}\right)^{1 / 3} \Psi\left(r_{h}\right)}{g^{\prime}\left(r_{h}\right)\left(1-\eta H\left(r_{h}\right)^{1 / 3} e^{\chi\left(r_{h}\right)} \Phi^{\prime 2}\left(r_{h}\right)\right)} .
$$

Near the boundary these fields asymptote to the following expressions

$$
\Phi=\mu-\frac{\rho}{r^{2}}+\ldots, \quad \Psi=\frac{\Psi_{-}}{r^{\lambda_{-}}}+\frac{\Psi_{+}}{r^{\lambda_{+}}}+\ldots \quad \chi \rightarrow 0, \quad g \rightarrow r^{2}+\ldots, \quad H \rightarrow 1+\ldots
$$

here $\lambda_{ \pm}=\frac{4 \pm \sqrt{16+4 m^{2}}}{2}$. 


\section{B Necessary formulas for the VGHS in 4-D AdS-Schwarzschild back- grounds}

We consider the following action

$$
\begin{aligned}
S= & \int \mathrm{d}^{4} x \sqrt{-g}\left[\frac{1}{2 \kappa^{2}}\left(R+\frac{6}{L^{2}}\right)-\frac{1}{4} F_{\mu \nu} F^{\mu \nu}-\frac{1}{2}\left|D_{\mu} \tilde{\Psi}\right|^{2}\right. \\
& \left.-\frac{1}{2} m^{2}|\tilde{\Psi}|^{2}-\frac{\eta}{2}\left|F_{\mu \nu} D^{\nu} \tilde{\Psi}\right|^{2}\right]
\end{aligned}
$$

As in section 2 , we rewrite $\tilde{\Psi}=\Psi e^{i \alpha}$ and the action becomes

$$
\begin{aligned}
S= & \int \mathrm{d}^{4} x \sqrt{-g}\left[\frac{1}{2 \kappa^{2}}\left(R+\frac{6}{L^{2}}\right)-\frac{1}{4} F_{\mu \nu} F^{\mu \nu}-\frac{\left(\partial_{\mu} \Psi\right)^{2}}{2}-\frac{m^{2} \Psi^{2}}{2}\right. \\
& \left.-\frac{\eta}{2} F_{\mu \nu} \partial^{\nu} \Psi F^{\mu \sigma} \partial_{\sigma} \Psi-\frac{\Psi^{2}(\partial \alpha-q A)^{2}}{2}-\frac{\eta}{2} \Psi^{2}\left(F^{\mu \nu}\left(\partial_{\nu} \alpha-q A_{\nu}\right)\right)^{2}\right]
\end{aligned}
$$

Now we replace $|\Psi|^{2}$ by two different analytic functions of $\Psi, G(\Psi)$ and $\mathrm{K}(\Psi)$, keeping in mind that the gauge invariance should be preserved. Thus we have our generalized action,

$$
\begin{aligned}
S= & \int \mathrm{d}^{4} x \sqrt{-g}\left[\frac{1}{2 \kappa^{2}}\left(R+\frac{6}{L^{2}}\right)-\frac{1}{4} F_{\mu \nu} F^{\mu \nu}-\frac{\left(\partial_{\mu} \Psi\right)^{2}}{2}-\frac{\eta}{2} F_{\mu \nu} \partial^{\nu} \Psi F^{\mu \sigma} \partial_{\sigma} \Psi\right. \\
& \left.-\frac{m^{2} \Psi^{2}}{2}-\frac{|\mathrm{G}(\Psi)|(\partial \alpha-q A)^{2}}{2}-\frac{\eta}{2}|\mathrm{~K}(\Psi)|\left(F^{\mu \nu}\left(\partial_{\nu} \alpha-q A_{\nu}\right)\right)^{2}\right]
\end{aligned}
$$

We take the background metric as,

$$
d s^{2}=-r^{2} f(r) e^{-\chi(r)} d t^{2}+\frac{d r^{2}}{r^{2} f(r)}+r^{2}\left(d x^{2}+d y^{2}\right)
$$

with the following ansatz

$$
\Psi=\Psi(r), \quad A=\Phi(r) d t .
$$

The Hawking temperature of the black hole is given by

$$
T_{H}=\left.\frac{r^{2} f^{\prime}(r) e^{-\chi(r) / 2}}{4 \pi}\right|_{r=r_{h}}
$$

where $f\left(r_{h}\right)=0$ defines the radius of the event horizon, $r_{h}$.

The equations of motion for the scalar field $\Psi(r)$ and the gauge field $\Phi(r)$ are,

$$
\begin{array}{r}
\Psi^{\prime \prime}\left(1-\eta \mathrm{e}^{\chi} \Phi^{\prime 2}\right)+\Psi^{\prime}\left(\frac{4}{r}+\frac{f^{\prime}}{f}-\frac{\chi^{\prime}}{2}-\frac{\eta \mathrm{e}^{\chi} f^{\prime} \Phi^{\prime 2}}{f}-\frac{\eta}{2} \mathrm{e}^{\chi} \Phi^{\prime 2} \chi^{\prime}-\frac{4 \eta \mathrm{e}^{\chi} \Phi^{\prime 2}}{r}\right. \\
\left.-2 \eta \mathrm{e}^{\chi} \Phi^{\prime} \Phi^{\prime \prime}\right)+\frac{\Phi^{2} \mathrm{e}^{\chi}}{2 r^{4} f^{2}} \frac{d \mathrm{G}(\Psi)}{d \Psi}-\frac{\eta \Phi^{2} \mathrm{e}^{2 \chi} \Phi^{\prime 2}}{2 r^{4} f^{2}} \frac{d \mathrm{~K}(\Psi)}{d \Psi}-\frac{m^{2} \Psi}{r^{2} f}=0 \\
\Phi^{\prime \prime}\left(1+\eta r^{2} f \Psi^{\prime 2}-\frac{\eta \mathrm{K}(\Psi) \Phi^{2} e^{\chi}}{r^{2} f}\right)+\Phi^{\prime}\left(\eta r^{2} f^{\prime} \Psi^{\prime 2}+\frac{1}{2} \eta r^{2} f \chi^{\prime} \Psi^{\prime 2}+4 \eta r f \Psi^{\prime 2}\right. \\
\left.+2 \eta r^{2} f \Psi^{\prime} \Psi^{\prime \prime}+\frac{\chi^{\prime}}{2}+\frac{2}{r}\right)+\eta \Phi^{2} \Phi^{\prime}\left(\frac{\mathrm{K}(\Psi) e^{\chi} f^{\prime}}{r^{2} f^{2}}-\frac{e^{\chi} \mathrm{K}(\Psi)^{\prime}}{r^{2} f}-\frac{3 \mathrm{~K}(\Psi) e^{\chi} \chi^{\prime}}{2 r^{2} f}\right) \\
-\Phi\left(\frac{\mathrm{G}(\Psi)}{r^{2} f}+\frac{\eta \mathrm{K}(\Psi) e^{\chi} \Phi^{\prime}(r)^{2}}{r^{2} f}\right)=0
\end{array}
$$


Moreover, the $(t, t)$ and $(r, r)$ components of Einstein equation are

$$
\begin{array}{r}
f^{\prime}+2 \kappa^{2} r\left(\frac{1}{4} \frac{\mathrm{G}(\Psi) \Phi^{2} e^{\chi}}{r^{4} f}-\frac{3 \eta}{4} \frac{\mathrm{K}(\Psi) \Phi^{2} e^{2 \chi} \Phi^{\prime 2}}{r^{4} f}+\frac{1}{4} \eta f e^{\chi} \Phi^{\prime 2} \Psi^{\prime 2}\right. \\
\left.+\frac{1}{4} f \Psi^{\prime 2}+\frac{1}{4} \frac{m^{2} \Psi^{2}}{r^{2}}+\frac{1}{4} \frac{e^{\chi} \Phi^{\prime 2}}{r^{2}}\right)-\frac{3}{r}+\frac{3 f}{r}=0 \\
\chi^{\prime}+2 \kappa^{2} r\left(\frac{\mathrm{G}(\Psi) \Phi^{2} e^{\chi}}{2 r^{4} f^{2}}-\frac{\eta \mathrm{K}(\Psi) \Phi^{2} e^{2 \chi} \Phi^{\prime 2}}{2 r^{4} f^{2}}-\frac{1}{2} \eta e^{\chi} \Phi^{\prime 2} \Psi^{\prime 2}+\frac{1}{2} \Psi^{\prime 2}\right)=0
\end{array}
$$

In the above equations again we have set $q=1$ and chosen the gauge $\alpha=0$. Also, the prime symbol indicates a derivative with respect to $r$.

We solve these four coupled differential equations using appropriate boundary conditions. At $r=r_{h}, \Phi=0$. Near the boundary they behave as

$$
\Phi=\mu-\frac{\rho}{r}+\ldots, \quad \Psi=\frac{\Psi_{-}}{r^{\lambda_{-}}}+\frac{\Psi_{+}}{r^{\lambda_{+}}}+\ldots, \quad \chi \rightarrow 0, \quad g \rightarrow r^{2}+\ldots
$$

We rewrite all the coupled equations in terms of $z=r_{h} / r$ and using the above boundary conditions we solve them numerically. Also, we will take the same values of $\xi, \theta$ and $\gamma$ as in [7], i.e, $\xi=0, \theta=4, \gamma=4$. At high temperature the condensate will vanish, so $\Psi=0$. The solution becomes

$$
\Psi=0, \quad \chi=0, \quad \Phi=\mu(1-z), \quad f=1-z^{3}\left(1+\frac{\kappa^{2} \mu^{2}}{2}\right)+\frac{z^{4} \kappa^{2} \mu^{2}}{2}
$$

The temperature is given by

$$
T=\frac{1}{4 \pi}\left(3-\frac{\kappa^{2} \mu^{2}}{2}\right) .
$$

Open Access. This article is distributed under the terms of the Creative Commons Attribution License (CC-BY 4.0), which permits any use, distribution and reproduction in any medium, provided the original author(s) and source are credited.

\section{References}

[1] J.M. Maldacena, The Large- $N$ limit of superconformal field theories and supergravity, Int. J. Theor. Phys. 38 (1999) 1113 [hep-th/9711200] [INSPIRE].

[2] S.A. Hartnoll, C.P. Herzog and G.T. Horowitz, Building a Holographic Superconductor, Phys. Rev. Lett. 101 (2008) 031601 [arXiv:0803.3295] [INSPIRE].

[3] T. Albash and C.V. Johnson, A Holographic Superconductor in an External Magnetic Field, JHEP 09 (2008) 121 [arXiv: 0804.3466] [INSPIRE].

[4] S.A. Hartnoll, C.P. Herzog and G.T. Horowitz, Holographic Superconductors, JHEP 12 (2008) 015 [arXiv:0810.1563] [INSPIRE].

[5] S. Ryu and T. Takayanagi, Holographic derivation of entanglement entropy from AdS/CFT, Phys. Rev. Lett. 96 (2006) 181602 [hep-th/0603001] [INSPIRE].

[6] T. Albash and C.V. Johnson, Holographic Studies of Entanglement Entropy in Superconductors, JHEP 05 (2012) 079 [arXiv: 1202.2605] [INSPIRE]. 
[7] A. Dey, S. Mahapatra and T. Sarkar, Generalized Holographic Superconductors with Higher Derivative Couplings, JHEP 06 (2014) 147 [arXiv:1404.2190] [INSPIRE].

[8] S. Franco, A. Garcia-Garcia and D. Rodriguez-Gomez, A General class of holographic superconductors, JHEP 04 (2010) 092 [arXiv:0906.1214] [INSPIRE].

[9] X.-M. Kuang, E. Papantonopoulos, G. Siopsis and B. Wang, Building a Holographic Superconductor with Higher-derivative Couplings, Phys. Rev. D 88 (2013) 086008 [arXiv: 1303.2575] [INSPIRE].

[10] S.S. Gubser, Breaking an Abelian gauge symmetry near a black hole horizon, Phys. Rev. D 78 (2008) 065034 [arXiv: 0801.2977] [INSPIRE].

[11] G.T. Horowitz and M.M. Roberts, Holographic Superconductors with Various Condensates, Phys. Rev. D 78 (2008) 126008 [arXiv:0810.1077] [InSPIRE].

[12] R. Gregory, S. Kanno and J. Soda, Holographic Superconductors with Higher Curvature Corrections, JHEP 10 (2009) 010 [arXiv:0907.3203] [INSPIRE].

[13] Q. Pan, B. Wang, E. Papantonopoulos, J. Oliveira and A.B. Pavan, Holographic Superconductors with various condensates in Einstein-Gauss-Bonnet gravity, Phys. Rev. D 81 (2010) 106007 [arXiv:0912.2475] [INSPIRE].

[14] R.-G. Cai, S. He, L. Li and L.-F. Li, Entanglement Entropy and Wilson Loop in Stúckelberg Holographic Insulator/Superconductor Model, JHEP 10 (2012) 107 [arXiv:1209.1019] [INSPIRE].

[15] S. Mahapatra, P. Phukon and T. Sarkar, Generalized Superconductors and Holographic Optics, JHEP 01 (2014) 135 [arXiv: 1305.6273] [INSPIRE].

[16] P. Yan and P. Q-Yuan, Stückelberg holographic superconductor models with backreactions, Commun. Theor. Phys. 59 (2013) 110.

[17] A. Bianchi, R. Movshovich, N. Oeschler, P. Gegenwart, F. Steglich et al., First order superconducting phase transition in CeCoIn(5), Phys. Rev. Lett. 89 (2002) 137002 [cond-mat/0203310] [INSPIRE].

[18] Physical Review Letters 110 (2013) 077003 [arXiv: 1212.4954].

[19] Y. Tanaka, A. Iyo, S. Itoh, K. Tokiwa, T. Nishio et al., Experimental observation of a first-order phase transition below the superconducting transition temperature in the multilayer cuprate superconductor $\mathrm{HgBa}_{2} \mathrm{Ca}_{4} \mathrm{Cu}_{5} \mathrm{O}_{y}$, J. Phys. Soc. Jap. 83 (2014) 074705 [arXiv: 1408.1445] [INSPIRE].

[20] N. Bobev, A. Kundu, K. Pilch and N.P. Warner, Minimal Holographic Superconductors from Maximal Supergravity, JHEP 03 (2012) 064 [arXiv:1110.3454] [INSPIRE].

[21] X.-M. Kuang, E. Papantonopoulos and B. Wang, Entanglement Entropy as a Probe of the Proximity Effect in Holographic Superconductors, JHEP 05 (2014) 130 [arXiv:1401.5720] [INSPIRE].

[22] H. Liu and M. Mezei, A Refinement of entanglement entropy and the number of degrees of freedom, JHEP 04 (2013) 162 [arXiv:1202.2070] [INSPIRE].

[23] P. Calabrese and J.L. Cardy, Entanglement entropy and quantum field theory, J. Stat. Mech. 0406 (2004) P06002 [hep-th/0405152] [INSPIRE].

[24] R.-G. Cai, L. Li, L.-F. Li and R.-K. Su, Entanglement Entropy in Holographic P-Wave Superconductor/Insulator Model, JHEP 06 (2013) 063 [arXiv:1303.4828] [INSPIRE]. 
[25] J. Bhattacharya, M. Nozaki, T. Takayanagi and T. Ugajin, Thermodynamical Property of Entanglement Entropy for Excited States, Phys. Rev. Lett. 110 (2013) 091602 [arXiv: 1212.1164] [INSPIRE].

[26] D.T. Son and A.O. Starinets, Minkowski space correlators in AdS/CFT correspondence: Recipe and applications, JHEP 09 (2002) 042 [hep-th/0205051] [INSPIRE].

[27] D. Allahbakhshi, M. Alishahiha and A. Naseh, Entanglement Thermodynamics, JHEP 08 (2013) 102 [arXiv:1305.2728] [INSPIRE].

[28] K. Maeda, M. Natsuume and T. Okamura, Dynamic critical phenomena in the AdS/CFT duality, Phys. Rev. D 78 (2008) 106007 [arXiv:0809.4074] [INSPIRE].

[29] V. Balasubramanian and P. Kraus, A Stress tensor for Anti-de Sitter gravity, Commun. Math. Phys. 208 (1999) 413 [hep-th/9902121] [INSPIRE].

[30] S. de Haro, S.N. Solodukhin and K. Skenderis, Holographic reconstruction of space-time and renormalization in the AdS/CFT correspondence, Commun. Math. Phys. 217 (2001) 595 [hep-th/0002230] [INSPIRE]. 